\title{
Dust emissivity variations in the Milky Way
}

\author{
D. Paradis ${ }^{1,2,3}$, J.-Ph. Bernard ${ }^{2,3}$, and C. Mény $y^{2,3}$ \\ 1 Spitzer Science Center, California Institute of Technology, Pasadena, CA 91125, USA \\ e-mail: paradis@ipac.caltech.edu \\ 2 Université de Toulouse, UPS, CESR, 9 Av. du Colonel Roche, 31028 Toulouse Cedex 9, France \\ 3 CNRS, UMR5187, 31028 Toulouse, France
}

Received 28 October 2008 / Accepted 3 July 2009

ABSTRACT

\begin{abstract}
Aims. Dust properties appear to vary according to the environment in which the dust evolves. Previous observational indications of these variations in the far-infrared (FIR) and submillimeter (submm) spectral range are scarce and limited to specific regions of the sky. To determine whether these results can be generalised to larger scales, we study the evolution in dust emissivities from the FIR to millimeter $(\mathrm{mm})$ wavelengths, in the atomic and molecular interstellar medium (ISM), along the Galactic plane towards the outer Galaxy.

Methods. We correlate the dust FIR to mm emission with the $\mathrm{HI}$ and $\mathrm{CO}$ emission, which are taken to trace the atomic and molecular phases, respectively. The study is carried out using the DIRBE data from 100 to $240 \mu \mathrm{m}$, the Archeops data from $550 \mu \mathrm{m}$ to $2.1 \mathrm{~mm}$, and the WMAP data at $3.2 \mathrm{~mm}$ (W band), in regions with Galactic latitude $|b| \leq 30^{\circ}$, over the Galactic longitude range $\left(75^{\circ}<1<198^{\circ}\right)$ observed with Archeops. We estimate the average dust temperature in each phase and divide the emission spectral energy distribution (SED) by a black body at this temperature to derive the emissivity profile. A detailed verification of the impact of the implied simplification, such as temperature mixing along the line of sight, is provided.

Results. In all regions studied, the emissivity spectra in both the atomic and molecular phases are steeper in the FIR $(\beta=2.4)$ than in the submm and $\mathrm{mm}(\beta=1.5)$. We find significant variations in the spectral shape of the dust emissivity as a function of the dust temperature in the molecular phase. Regions of similar dust temperature in the molecular and atomic gas exhibit similar emissivity spectra. Regions where the dust is significantly colder in the molecular phase show a significant increase in emissivity for the range $100-550 \mu \mathrm{m}$. We exclude the possibility of this effect being an artifact of our temperature determination or the assumptions made. This result supports the hypothesis of grain coagulation in these regions, confirming results obtained over small fractions of the sky in previous studies and allowing us to expand these results to the cold molecular environments in general of the outer MW. Possible reasons for the observed emissivity increase in the molecular phase that vanishes in the $\mathrm{mm}$ range are discussed by comparison with dust models, involving dust aggregation and solid state physics processes specific to amorphous material. We note that it is the first time that these effects have been demonstrated by direct measurement of the emissivity, while previous studies were based only on thermal arguments.
\end{abstract}

Key words. ISM: dust, extinction - infrared: ISM - submillimeter

\section{Introduction}

Measuring the dust emissivity is important to inferring the nature of dust from its emission and also determining the dust heating from its observed temperature. In addition, variations in the dust emissivity may seriously affect the mass estimates inferred from FIR to mm observations. Dust emissivity and its possible variations with wavelength or temperature are also critical for separating astrophysical foreground emission from the cosmic microwave background (CMB). However, deriving dust emissivity is generally a difficult task because it relies on both estimating the dust temperature and the gas column density associated with the emitting dust. Big dust Grains (BG, see Désert et al. 1990, for a description of each dust component) are in thermal equilibrium with the interstellar radiation field (ISRF). Their emission is close to that of a gray-body with an equilibrium temperature near 17.5 K in the diffuse ISM (Boulanger et al. 1996; Lagache et al. 1998), with a maximum in the far-infrared. The emission

\footnotetext{
* Appendix A is only available in electronic form at http://www . aanda.org
}

spectrum, assuming a fixed dust abundance and a single grain size, follows

$I_{v}(\lambda)=\epsilon_{v}(\lambda) B_{v}\left(\lambda, T_{\mathrm{d}}\right) N_{\mathrm{H}}$,

where $I_{v}(\lambda)$ is the specific intensity or brightness (energy flux per unit frequency and solid angle), $B_{v}$ is the Planck function, $T_{\mathrm{d}}$ is the equilibrium temperature of grains, $N_{\mathrm{H}}$ is the total column density (number of hydrogen atoms per unit area), and $\epsilon_{v}(\lambda)$ is the wavelength-dependent dust grain emissivity.

Although, in principle, the emissivity may depend on wavelength and temperature (e.g., Mény et al. 2007), it has been customary to assume no temperature dependence and a power law distribution with frequency,

$\epsilon_{v}(\lambda)=X_{\mathrm{d}} \epsilon_{0}\left(\frac{\lambda}{\lambda_{0}}\right)^{-\beta}$

where $X_{\mathrm{d}}$ is the dust to gas mass ratio, $\epsilon_{0}$ is the emissivity at wavelength $\lambda_{0}$, and $\beta$ is the emissivity spectral index. The spectral index is usually assumed to equal 2 , which is correct for crystalline grains. However, dust grains are known to be amorphous in the diffuse ISM (Kemper et al. 2004) and this could 
have an impact on the emissivity. Grains are probably affected by different physical processes depending on the environment. Their properties are likely to vary according to the density of the medium. Different studies have highlighted changes in the grain properties in the dense and cold medium, such as the depletion of chemical elements (Caselli et al. 1999; Bacmann et al. 2002), that imply that condensation of gaseous species is occuring on the grain surface.

A decrease in the IRAS $I_{60} / I_{100}$ ratio in several isolated molecular clouds has also been noted (e.g., Laureijs et al. 1991; Abergel et al. 1994, 1995), whereas this ratio seems to be constant in diffuse regions. This could be interpreted as a decrease of very small grain (VSG) abundance with respect to that of the BGs in dense molecular clouds. Bernard et al. (1999) analysed the FIR dust emission from a molecular cirrus in Polaris, using the PRONAOS balloon-borne experiment data and found a larger decrease in the BG equilibrium temperature than predicted from the decrease in the ISRF intensity in the cloud. The same region showed an obvious deficit in $60 \mu \mathrm{m}$ emission. They attributed the change in the particular dust properties of dense environments.

Stepnik et al. (2003) attempted to explain the origin of the decrease in VSG abundance and the drop in the BG temperature in dense environments. They measured a BG temperature of $16.8 \mathrm{~K}$ outside a filament in Taurus, and of $12 \mathrm{~K}$ at the centre of the filament, using the PRONAOS and the IRAS data. They showed that a model that assumed the standard properties of dust, without any spatial variations in the grain properties, was unable to reproduce the observations. The attenuation of the radiation field in the cloud was also unable to explain the shape of the observed emission profiles. They proposed that the aggregation of large dust grains and most of the VSGs in fractal assemblies would explain both the observed $60 \mu \mathrm{m}$ emission deficit and the unusually cold BG temperature. They showed that aggregation would lead to an increase in the BG emissivities by a factor of 3-4, and that between 80 and $100 \%$ of the VSGs should participate in the aggregation process. The best-fit model showed that the VSG and BG aggregation occurs at an extinction $A v>2.1 \mathrm{mag}$ and at densities higher than $n_{\mathrm{H}}=3 \mathrm{H} / \mathrm{cm}^{3}$. However, the emissivity increase in dense environments remains indirect evidence of change in the emissivity, since $T_{\mathrm{d}}$ is lower than expected for the corresponding ISRF. This effect has never been illustrated by a direct determination of the dust emissivity, mainly because both the dust temperature and $N_{\mathrm{H}}$ are difficult to measure.

Cambrésy et al. (2001a) analysed the DIRBE emission of the Polaris complex over $20 \mathrm{deg} 2$, to determine the dust properties. They decomposed the emission into a warm and a cold component constrained using the $I_{60} / I_{100}$ IRAS intensity ratio. They determined the extinction map of this complex in the visible, by using star counts in the $V$ band (Cambrésy 1999). They calculated the submillimeter emissivity of each component, normalised to the visible extinction, and found that the emissivity is 4 times higher in the cold region of the complex than in the warm region. This result matches the emissivity increase derived to explain the low dust temperature by Stepnik et al. (2003), and was based on the determination of the submm to visible dust opacity ratio, which traces the optical properties of the grain material, independent of the gas column density.

Two hypotheses have been proposed to explain the observations: the formation of ice mantles (Laureijs et al. 1991, 1996) and/or grain aggregation (Draine \& Anderson 1985; Tielens 1989). The $60 \mu \mathrm{m}$ emission deficit in dense regions can be attributed to a large majority of VSGs participating in the aggregation process, and the VSGs are almost totally accreted onto the aggregates, in the inner regions of the cloud (Stepnik et al. 2003). The VSGs are then connected to the aggregate, which acts as a thermostat. Their temperature then stops fluctuating and their contribution to the mid-IR greatly decreases, which explains their weak IRAS $60 \mu \mathrm{m}$ emission. Calculations, for instance using the discrete dipole approximation (DDA) method, of optical properties of aggregates (Bazell \& Dwek 1990) show that BG aggregates are more efficient emitters than a collection of individual grains. This increased emissivity leads to more efficient cooling for a given ISRF intensity and therefore explains the lower temperature observed. The calculations indicate that the aggregate emissivity should progressively deviate from that of the individual grains in the IR (around $20 \mu \mathrm{m}$ ), and remain parallel to it at longer wavelengths, in particular over the entire submillimeter and millimeter range.

The adjunction of VSGs in the aggregate seems to have little effect on FIR/mm properties and simply provides a slightly higher absorptivity of the aggregate in the near-IR (Stepnik 2001a). Calculations by Stepnik et al. (2003) show that a total number of at least 20 individual BG in each aggregate is necessary to provide the required emissivity increase. However, for the aggregation process to be efficient, the individual grains should probably be covered with an ice mantle, to increase the sticking efficiency.

The parameter $\epsilon_{v}$ in Eq. (1) is consistent with the definition used in Boulanger et al. (1996). The emissivity $\epsilon_{v}$ is related to the absorption efficiency $Q_{\mathrm{abs}}$, which is directly related to the refractive index of the material composing the grain by

$\epsilon_{v}(\lambda)=\pi a^{2} Q_{\mathrm{abs}}(\lambda, a) X_{\mathrm{d}}$

where $a$ is the grain radius. Therefore, for a single grain size, the wavelength dependence of $\epsilon_{v}$ and $Q_{\text {abs }}$ are the same. The purpose of this paper is to determine the spectral dependence of the dust absorption cross section in the atomic and molecular phases of the ISM and to search systematically for evidence of emissivity changes in the dense molecular medium, to check whether the results obtained for a limited number of isolated clouds can be generalised. In the following, we use Eq. (1) to derive the wavelength dependence of the dust emissivity, by simply dividing the observed emission $I_{v}(\lambda)$ by $B_{v}\left(\lambda, T_{\mathrm{d}}\right) N_{\mathrm{H}}$, for a single temperature $T_{\mathrm{d}}$ for each ISM phase. However, we note that, for a realistic grain population, Eq. (1) must be integrated over the grain size distribution. Since the dust temperature depends on the dust grain size for a given radiation field, the black-body term in Eq. (1) cannot be factorised out from the integral. Similarly, most lines of sight (LOS) must exhibit variations in the radiation field, leading to a mixture of dust temperature along the LOS. As a consequence, Eq. (1) only measures an "effective emissivity", whose wavelength dependence may differ from that of the true emissivity of the material constituent of the grain. In Sect. 7, we show however that our approach is valid over the range of dust temperature and wavelengths considered here, and that the effective emissivity precisely recovers the wavelength dependence of the true material emissivity in the presence of both a realistic grain size distribution and a realistic mixture of the radiation field along the LOS. In the following, we therefore simply refer to $\epsilon_{v}$ as the dust emissivity, and use Eq. (1) to derive the emissivity directly.

In Sect. 2, we present the data that we used for our study and in Sect. 3 we define the emission to column density conversion factors used. In Sect. 4, we describe the correlation procedure between dust emission and gas tracers. In Sect. 5, we explain how we compute the grain emissivity. The results of this study 
and the verification of our hypothesis of a single temperature along the LOS are presented in Sects. 6 and 7. Sections 8 and 9 are devoted to our discussion and conclusions.

\section{Data}

\subsection{FIR data}

The Diffuse Infrared Background Experiment (DIRBE) was an infrared photometer onboard the COBE satellite (launched in 1989) to measure the diffuse infrared and microwave radiation from the early universe. It observed the entire sky at 10 different wavelengths between 1 and $240 \mu \mathrm{m}$, with a 40' instantaneous angular resolution. However, the asymmetric beam of the DIRBE instrument convolved with the spinning of the instrument produced an effective beam of $\simeq 1^{\circ}$ in the yearly averaged products (Cambrésy et al. 2001b). For our study, we only consider data in the far-infrared at 100,140 , and $240 \mu \mathrm{m}$, since emission at shorter wavelengths contains a large contribution from thermally fluctuating VSGs and polycyclic aromatic hydrocarbons (PAH). The DIRBE data at these wavelengths were calibrated with models for Uranus $(100 \mu \mathrm{m})$ and Jupiter (140 and $240 \mu \mathrm{m})$ together with in-flight beam shape measurements. This calibration matched that of the FIRAS instrument, which incorporated an absolute calibrator, within a small correction factor, which we did not apply in this study since it is smaller than our uncertainties (Fixen et al. 1997).

Archeops was a balloon-borne experiment dedicated to the measure of the temperature fluctuations in the CMB. The focal plane instrument, a multi-band photometer, worked in four bands centered on $550 \mu \mathrm{m}, 850 \mu \mathrm{m}, 1.4 \mathrm{~mm}$, and $2.1 \mathrm{~mm}$. Archeops had an angular resolution of $8^{\prime}$ (see Benoit et al. 2002 , for a full description of the instrument). We use data obtained during the last flight of the instrument from Kiruna in February 2002, which covered about $30 \%$ of the sky. We note that we limit our analysis to the region surveyed by Archeops, which is given by the range of Galactic longitudes $75^{\circ} \leq 1 \leq$ $198^{\circ}$, in the second and third Galactic quadrants. The Archeops data were calibrated against the FIRAS maps, at $550 \mu \mathrm{m}$ and $850 \mu \mathrm{m}$, and with respect to the CMB dipole at $1.4 \mathrm{~mm}$ and $2.1 \mathrm{~mm}$. We note that the calibration of the Archeops data should be accurate at all wavelengths, since it relies on the FIRAS maps at high frequencies, which are absolutely calibrated to $3 \%$ (Fixsen et al. 1994), and on the CMB dipole at low frequencies, whose brightness is accurately known. In addition, the Archeops cosmology results derived from the low frequency channels have been shown to be consistent with those of the WMAP satellite at the 7\% accuracy (Tristram et al. 2005). Details of the Archeops data processing are given in MaciasPerez et al. (2007). We note that the Archeops channel at $550 \mu \mathrm{m}$ has residual stripes, because only one detector was available at that frequency.

The Wilkinson Microwave Anisotropy Probe (WMAP) measured the emission over the entire sky in the microwave range (3.2-13 $\mathrm{mm})$, and provided accurate maps of the CMB fluctuations. The WMAP data, with a $13^{\prime}$ beam, is currently the instrument of the highest angular resolution covering the entire sky in this wavelength domain. Here, we use only the $\mathrm{W}$ band data $(3.2 \mathrm{~mm})$, because the other bands are often dominated by gas emission, such as free-free and synchrotron, or by the anomalous foreground emission (see Draine \& Lazarian 1998; Bennett et al. 2003), presumably because of small spinning particles. The calibration of the WMAP data was completed using the CMB dipole amplitude (see Hinshaw et al. 2003).

\subsection{Gas tracers}

We use the Leiden/Dwingeloo survey by Hartmann \& Burton (1996) for the HI data, with sky observations above $-30^{\circ}$ of Galactic latitude, obtained with the $25 \mathrm{~m}$ Dwingeloo telescope, whose angular resolution is 36' (see Hartmann 1994, for more details).

We use ${ }^{12} \mathrm{CO}(J=1-0)$ data compiled by Dame et al. (2001). These observations were obtained along the Galactic plane in a Galactic latitude range from $4^{\circ}$ to $10^{\circ}$, with an angular resolution of $7.5^{\prime}$, and some observations of large clouds at higher latitude, with an angular resolution of $15^{\prime}$. The spatial coverage is about $45 \%$ of the sky.

All data were projected onto the HEALPix pixelisation scheme (Hierarchical Equal Area isoLatitude Pixelisation) ${ }^{1}$ with a nside $=128$, corresponding to a pixel size of $0.45^{\circ}$. For the Archeops and WMAP first release data, we use the published maps, and for $\mathrm{HI}$ and $\mathrm{CO}$, we use the maps available on the WMAP lambda web site ${ }^{2}$. For the DIRBE data, we used our own resampling method developed in the context of the ancillary data for the Planck mission, computing the pixel intersection between the original DIRBE maps in the sixcube format and the HEALPix pixelisation. The method preserves the photometry without significantly affecting the angular resolution. Since the Archeops data were filtered to subtract slow drifts, we simulated fake data stream with all data described in this section, according to the actual Archeops scanning strategy. Those data time-lines were treated in a similar way to the Archeops ones, in particular by including the low frequency filtering applied to the Archeops data, and were then reprojected into sky maps. Using the standard HEALPix tools, all the data were corrected to the DIRBE angular resolution $\left(1^{\circ}\right)$, by convolution with a Gaussian kernel of an appropriate size in relation to the original resolution of each dataset.

\section{Conversion factors}

Assuming that the gas is optically thin, the hydrogen column density can be deduced from the integrated intensity of the $\mathrm{HI}$ emission at $21 \mathrm{~cm}\left(W_{\mathrm{HI}}\right)$ using the relation

$N_{\mathrm{HI}}=X_{\mathrm{HI}} W_{\mathrm{HI}}$,

where $X_{\mathrm{HI}}$ is the $\mathrm{HI}$ integrated intensity to column density conversion factor, assumed to be equal to $1.82 \times$ $10^{18} \mathrm{H}_{2} / \mathrm{cm}^{2} /(\mathrm{Kkm} / \mathrm{s}$ ) (Spitzer 1978). The $\mathrm{CO}$ to molecular conversion factor is given by $X_{\mathrm{CO}}=\frac{N_{\mathrm{H}_{2}}}{W_{\mathrm{CO}}}$, where $W_{\mathrm{CO}}$ is the integrated $\mathrm{CO}$ emission, and we assume a fixed value of $X_{\mathrm{CO}}$, which is defined to be the standard Galactic value proposed by Strong et al. (1988)

$X_{\mathrm{CO}}=2.3 \times 10^{20} \mathrm{H}_{2} / \mathrm{cm}^{2} /(\mathrm{Kkm} / \mathrm{s})$.

This value was inferred from $\gamma$-ray measurements over the entire Galactic plane excluding the Galactic center region.

\section{Correlation between IR and gas tracers}

We considered only regions along the Galactic plane with $|b| \leq 30^{\circ}$ and pixels with sufficient $\mathrm{HI}$ or $\mathrm{CO}$ emission

\footnotetext{
${ }_{1}$ HEALPix pixelisation distributes $12 \times \mathrm{Nside}^{2}$ points as uniformly as possible on a sphere surface, knowing that these points are divided into (4Nside-1) parallel on latitudes and are fairly spaced on longitudes on each one of these parallels. See http://healpix.jpl.nasa.gov/ 2 (http://lambda.gsfc.nasa.gov/)
} 
$\left(W_{\mathrm{HI}}>1000 \mathrm{~K} / \mathrm{km} / \mathrm{s}\right.$ and $\left.W_{\mathrm{CO}}>0.5 \mathrm{~K} / \mathrm{km} / \mathrm{s}\right)$. To assess the possible contribution from free-free, synchrotron, and spinning dust emission on the IR emission in the WMAP W band, we used the Planck Sky Model ${ }^{3}$ (Delabrouille et al. 2009). The model indicates that the free-free emission is the main contribution in this band and generates about $30 \%$ of the IR emission. Its contribution was subtracted from the $\mathrm{W}$ band emission, using the free-free map predicted by the Planck Sky Model. Other contaminations (synchrotron, spinning dust) are negligible in this band $(<3 \%)$, according to the same model. The contributions from free-free, synchrotron, and spinning dust are insignificant in other data used in this study.

We performed correlations between the infrared emission and gas tracers, in a set of rectangular regions covering the area of interest. Individual regions have sizes $\Delta l \times \Delta b=6^{\circ} \times 4^{\circ}$, distributed across a regular grid of Galactic coordinates every $3^{\circ}$ in longitude and $2^{\circ}$ in latitude. In each region and each map, we subtracted a background value computed as the median over a common background area, defined to be the faintest half of the HI data. In this way, we ensured that the correlation produces a null IR emission for a null column density. This step also removes any possible residual contribution from both the Zodiacal light and the cosmic infrared background. We then determined the best-fit linear correlation between the FIR emission and gas tracers using

$I_{v}(\lambda)=\frac{a_{v}(\lambda)}{X_{\mathrm{HI}}} N_{\mathrm{H}}^{\mathrm{HI}}+\frac{b_{v}(\lambda)}{2 X_{\mathrm{CO}}} N_{\mathrm{H}}^{\mathrm{CO}}+c_{v}(\lambda)$,

where $I_{v}$ is the IR emission brightness at wavelength $\lambda, N_{\mathrm{H}}^{\mathrm{HI}}$ and $N_{\mathrm{H}}^{\mathrm{CO}}$ are the hydrogen column density in the atomic and molecular phases, respectively, and $c_{v}$ is a constant. The constant term is usually small but could account for the contribution of an additional gas component unaccounted for in our description, such as diffuse ionized gas, provided it is partially decorrelated from the atomic and molecular gas distribution. We performed a $\chi^{2}$ minimization searching for $a_{v}$ and $b_{v}$ at each IR wavelength and for each region using the interactive data language (IDL) linear regression function "regress".

In a second step, we rejected regions of smaller than 15 pixels to maintain a sufficient number of pixels to perform the correlations. The number of pixels in each region is given in tables in the Appendix. With this selection, we removed $15 \%$ of the regions. In a third step, we removed regions with clearly unphysical values of the dust temperatures $(>1000 \mathrm{~K})$. For temperatures inferred from the spectral shape of $a_{v}$ and $b_{v}$ between 100 and $550 \mu \mathrm{m}$ (see Sect. 5 for more explanation), these unphysical values imply that at least one of the correlation coefficients $a_{v}$ or $b_{v}$ in this wavelength range is still affected by uncorrected instrumental effects, mostly residual stripes in the Archeops $550 \mu \mathrm{m}$ channel. This selection removed $27 \%$ of the remaining regions.

\section{Grain emissivity determination}

\subsection{Method}

Using Eqs. (1) and (6), and assuming a single temperature of dust in the atomic $\left(T_{\mathrm{d}}^{\mathrm{HI}}\right)$ and the molecular $\left(T_{\mathrm{d}}^{\mathrm{CO}}\right)$ phases, the emissivity of the dust associated with each phase can be written

$\epsilon_{v}^{\mathrm{HI}}(\lambda)=\frac{a_{v}(\lambda)}{B_{v}\left(T_{\mathrm{d}}^{\mathrm{HI}}\right)} \frac{1}{X_{\mathrm{HI}}}$,

3 see http://wWw.apc.univ-paris7.fr/APC_CS/Recherche/ Adamis/PSM/psky . php and

$\epsilon_{v}^{\mathrm{CO}}(\lambda)=\frac{b_{v}(\lambda)}{B_{v}\left(T_{\mathrm{d}}^{\mathrm{CO}}\right)} \frac{1}{2 X_{\mathrm{CO}}}$,

respectively. The values of the dust temperatures in the two phases can be obtained by fitting the SED of the correlation coefficients $a_{v}$ and $b_{v}$, which is equivalent, for a given column density, to the emission spectrum of dust associated with the atomic and molecular phases, respectively. We fitted the above SEDs by searching simultaneously for the best-fit average temperature and dust emissivity index for each phase, using the correlation coefficients at 100, 140, 240, and $550 \mu \mathrm{m}$. Uncertainties in the emissivity values of each region are computed using

$\Delta \epsilon_{v} / \epsilon_{v}=\frac{\Delta B_{v}(T)}{B_{v}(T)}+\frac{\Delta I_{v}}{I_{v}}$,

where the uncertainties on the intensity for the atomic and molecular phase are given by $\Delta I_{v, \mathrm{HI}}=\frac{\Delta a_{v}}{X_{\mathrm{HI}}}$ and $\Delta I_{v, \mathrm{CO}}=\frac{\Delta b_{v}}{2 X_{\mathrm{CO}}}$, respectively. Errors in the $a_{v}$ and $b_{v}$ parameters correspond to the 1- $\sigma$ standard deviation computed over all valid pixels of the region. Given the Planck function

$B_{v}(T)=\frac{2 h v^{3}}{c^{2}} \frac{1}{\mathrm{e}^{\frac{h \nu}{k T}}-1}$

the relative error in $B_{v}(T)\left(\Delta B_{v}(T)\right)$ is related to the error in $T$ $(\Delta T)$ by

$\frac{\Delta B_{v}(T)}{B_{v}(T)}=\frac{\frac{h v}{k T} \mathrm{e}^{\frac{h v}{k T}}}{\mathrm{e}^{\frac{h v}{k T}}-1} \frac{\Delta T}{T}$.

Errors in temperature used to derive the error in the Planck function are determined using a bootstrap method, when fitting for the temperature and spectral index. We note that the temperatures are mostly constrained by values close to the peak of the emission (i.e., 100, 140, and $240 \mu \mathrm{m}$ ). However, uncertainties in the derived temperature will strongly affect the emissivity values close the peak, because we divide by the black-body function, a non-linear function of the temperature near the peak. Therefore, the uncertainty in the temperature is the main source of uncertainty in this study. Overestimating the dust temperature by $2 \mathrm{~K}$ leads to an overestimate of $B_{v}$ and therefore an underestimate of the dust emissivity by as much as $150 \%$ and $70 \%$ at $100 \mu \mathrm{m}$ and $240 \mu \mathrm{m}$, respectively, for an average dust temperature of $16 \mathrm{~K}$. For $\Delta T=0.5 \mathrm{~K}$, these figures become $65 \%$ and $35 \%$, respectively.

All data used in this study follow a given flux convention (e.g., $v I_{v}=$ constant for DIRBE data), which allows us to compute unambiguously the total power received in the instrument photometric band, regardless of the energy distribution in the band. However, the true brightness at the reference frequency depends on the emission spectral shape, which is unknown until a fit using a model has converged. We therefore corrected the emissivity derived above by dividing by the appropriate color correction factor, computed using the filter transmission and flux convention of each instrument used. This was done in an iterative way, starting from color correction factors derived from a dust model, and iterating for the true shape of each SED, until convergence was reached.

\subsection{Classification according to dust temperature}

Dust in dense molecular clouds is expected to be colder than in the surrounding atomic material, as long as no significant 
star formation is occurring in the cloud. However, massive star formation can significantly heat the dust in molecular clouds. In addition, star formation may increase significantly the turbulence in the star-forming molecular medium, which in turn, could modify the dust emission properties. To search for these potential variations, we classify the regions according to 3 categories based upon the dust temperature derived for dust in the atomic phase $\left(T_{\mathrm{d}}^{\mathrm{HI}}\right)$ and in the molecular phase $\left(T_{\mathrm{d}}^{\mathrm{CO}}\right)$ :

- regular: atomic and molecular medium with similar dust temperatures defined as $T_{\mathrm{d}}^{\mathrm{CO}}<T_{\mathrm{d}}^{\mathrm{HI}}<T_{\mathrm{d}}^{\mathrm{CO}}+0.6 \mathrm{~K}$. This case allows us to compare the dust emissivity in the two environments in regions where the ISRF intensity is similar in both phases. This category accounts for 18 regions in our sample;

- colder CO: dust in the molecular phase significantly colder than in the atomic phase: $T_{\mathrm{d}}^{\mathrm{CO}}+2.5 \mathrm{~K}<T_{\mathrm{d}}^{\mathrm{HI}}<T_{\mathrm{d}}^{\mathrm{CO}}+4 \mathrm{~K}$. In this case, the colder dust temperature in the molecular phase is indicative of a lower ISRF intensity in the cloud, most likely indicating dense molecular environment with little star formation. This case is the most relevant to the search for emissivity variations linked to the formation of dust aggregates, since so far, these have been found in such regions (see e.g., Bernard et al. 1999; Stepnik et al. 2003). This category accounts for 45 regions in our sample;

- warmer CO: dust in the atomic phase significantly colder than in the molecular phase: $T_{\mathrm{d}}^{\mathrm{HI}}+2 \mathrm{~K}<T_{\mathrm{d}}^{\mathrm{CO}}<T_{\mathrm{d}}^{\mathrm{HI}}+4 \mathrm{~K}$. This category should incorporate most of the massive starforming regions. The lower temperature (here $T_{\mathrm{d}}^{\mathrm{HI}}$ ) is lower than in the previous case 2 in order to include enough regions. This category accounts for 25 regions in our sample.

Figure 1 shows the location of all studied regions for the three temperature cases. Temperature histograms are shown in Fig. 2, for each case and phase. Although the cases are selected based on the relative temperature between the two phases, it can be seen that they also select absolute temperature behaviors, since the absolute values of the dust temperatures are roughly reversed in case 2 and $3\left(T_{\mathrm{HI}}^{\mathrm{Case}} \simeq T_{\mathrm{CO}}^{\mathrm{Case} 3} \simeq 17 \mathrm{~K}\right.$ and $T_{\mathrm{CO}}^{\mathrm{Case} 2} \simeq T_{\mathrm{HI}}^{\mathrm{Case} 3} \simeq$ $14 \mathrm{~K}$ ). Appendix (Tables 7 and 8) indicates for each case and studied region, the number of pixels considered, the correlation factors obtained as well as the dust temperature values, and the emissivity spectral index in both the FIR and the submm range.

\section{Results}

Figure 3 shows the SEDs of the median emissivity derived from the correlation values, for the atomic and molecular phases, for each of the cases described in Sect. 5.2. The corresponding values are also given in Table 1.

The emissivity values shown in Fig. 3 were not normalised in any other way than assuming the fixed conversion factors described in Sect. 3. In Fig. 3, we also show the emissivity value derived from the FIRAS data by Boulanger et al. (1996) for the atomic diffuse ISM in the solar neighbourhood at $\lambda=250 \mu \mathrm{m}$, assuming $\beta=2$. It can be seen that the emissivity values derived here are in rough agreement with that value. We note however that the atomic emissivities that we derived are somewhat higher than in the solar neighbourhood. We also note that there is a continuity in the emissivity SED between the WMAP W band and the Archeops range indicating that there is no obvious calibration difference between the two experiments, which are both calibrated to the $\mathrm{CMB}$ dipole in this range.

The scaling of emissivity values for the molecular phase can be modified by assuming a different $X_{\mathrm{CO}}$ factor. The $X_{\mathrm{CO}}$ factor is

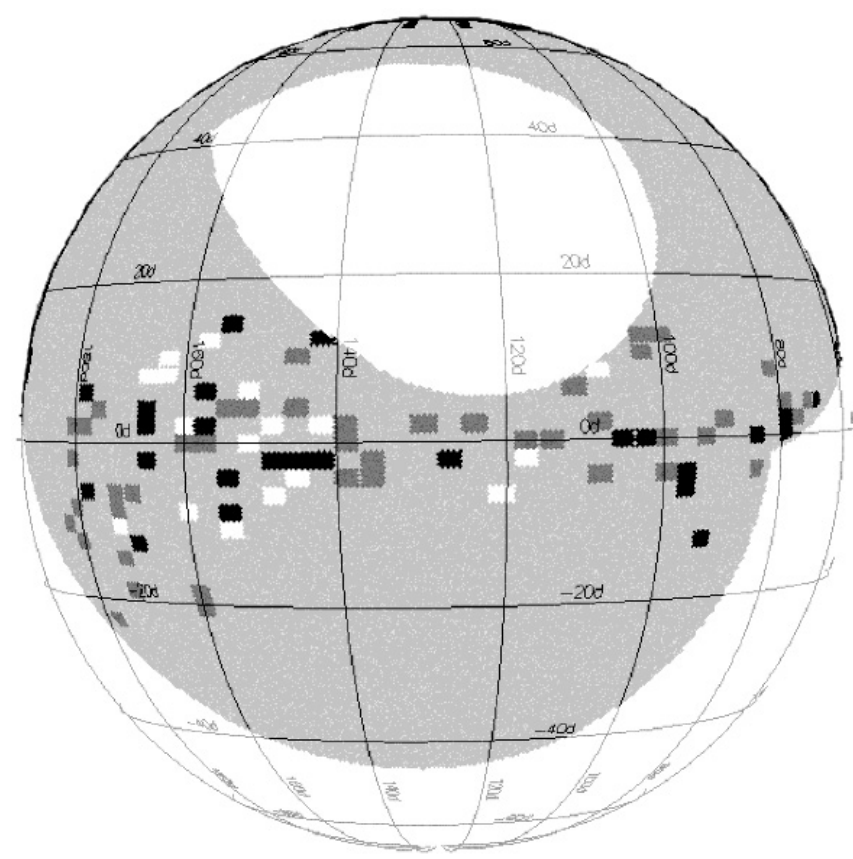

Fig. 1. Studied regions for each temperature case: case 1 (regular) in white, case 2 (colder CO) in grey and case 3 (warmer CO) in black. The sky region covered by the Archeops data is also delineated. The coordinate grid shown is in Galactic coordinates. The map shows half the sky and is centered roughly towards the Galactic anticenter.

difficult to determine. It is expected to vary with various factors such as the metallicity and the chemistry of the ISM, and could therefore change from cloud to cloud (e.g., Magnani \& Onello 1995). In Fig. 4, we show the same emissivity SEDs, where we have arbitrarily chosen the $X_{\mathrm{CO}}$ value so that the atomic and molecular emissivities match at the longest wavelengths. For each case, the derived $X_{\mathrm{CO}}$ values are given in the plot. Figure 4 more clearly shows the variations in the spectral shape. From that figure it is apparent that in case 1 and 3, the atomic and molecular medium emissivity SEDs are almost parallel, while in case 2 (colder CO), the molecular emissivity SED is significantly steeper than the atomic one in the FIR range. We note however, that if the absolute level of the emissivities in the molecular phase can vary according to the assumed $X_{\mathrm{CO}}$ value, the spectral shape of the emissivity SED and therefore the emissivity slope $(\beta)$ will remain unchanged.

In Fig. 4 , we also show power laws with $\beta=2.5, \beta=2$ and $\beta=1.5$ normalised at $100 \mu \mathrm{m}$ for the atomic phase. Although all emissivity SEDs globally show the expected decrease with wavelength, it can be seen that, in all 3 cases, the emissivity SED is steeper in the FIR (DIRBE data) and flattens significantly in the submillimeter above $\lambda>500 \mu \mathrm{m}$ (Archeops and WMAP data). Tables 2 and 3 summarise the median values of the emissivity spectral index at all wavelengths in the DIRBE and Archeops wavelength ranges, respectively. These values were inferred from a linear adjustment of the emissivity SEDs. Uncertainties correspond to $1-\sigma$. It can be seen that the emissivity index is generally higher than $\beta^{\text {fir }} \simeq 2$ in the FIR corresponding to a steep emissivity decrease with wavelength, and is about $\beta^{\text {submm }} \simeq 1.5$ in the submm and mm domain, corresponding to a flatter spectrum. Apart from this common behavior, there are also significant differences between the 3 temperature cases, which are discussed below. 

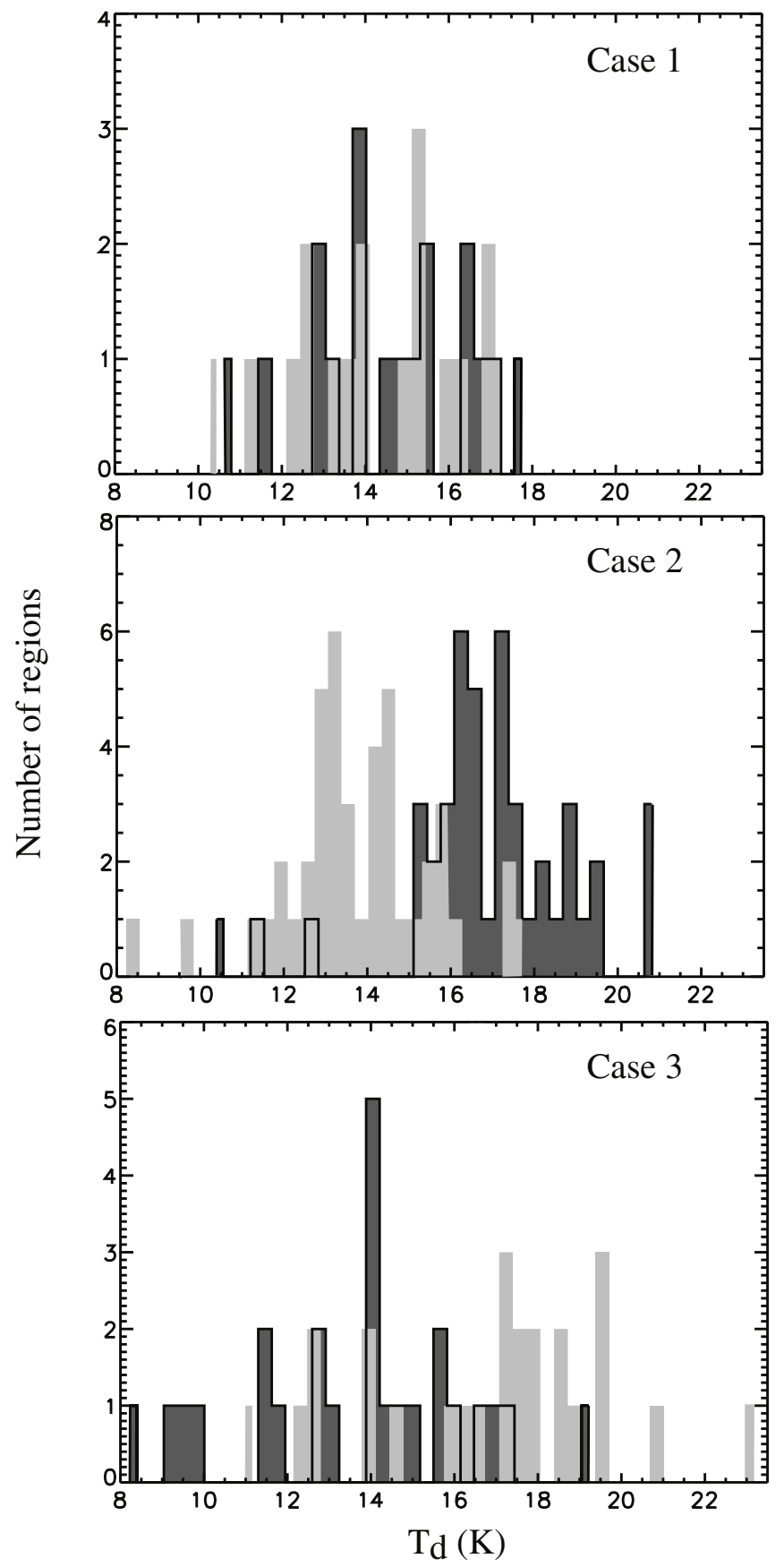

Fig. 2. Dust temperature histograms for each selected case: the atomic and molecular phases are shown in dark grey with black contours and light grey, respectively.

\subsection{Case 1: regular}

In this case, the emissivity SED of the dust associated with the atomic and molecular phases are almost parallel, and both clearly experience the same slope change between the DIRBE and the Archeops wavelengths. This similarity probably indicates that, in this case, the dust emission properties associated with the molecular and atomic phases are the same.

In addition, the absolute emissivity values in the two phases are relatively close. This indicates that our adopted $X_{\mathrm{CO}}$ value is reasonable in that case. There is no reason in principle that the dust abundance $X_{\mathrm{d}}$ could differ between the two phases. Therefore, an incorrect estimate of the $X_{\mathrm{CO}}$ parameter would

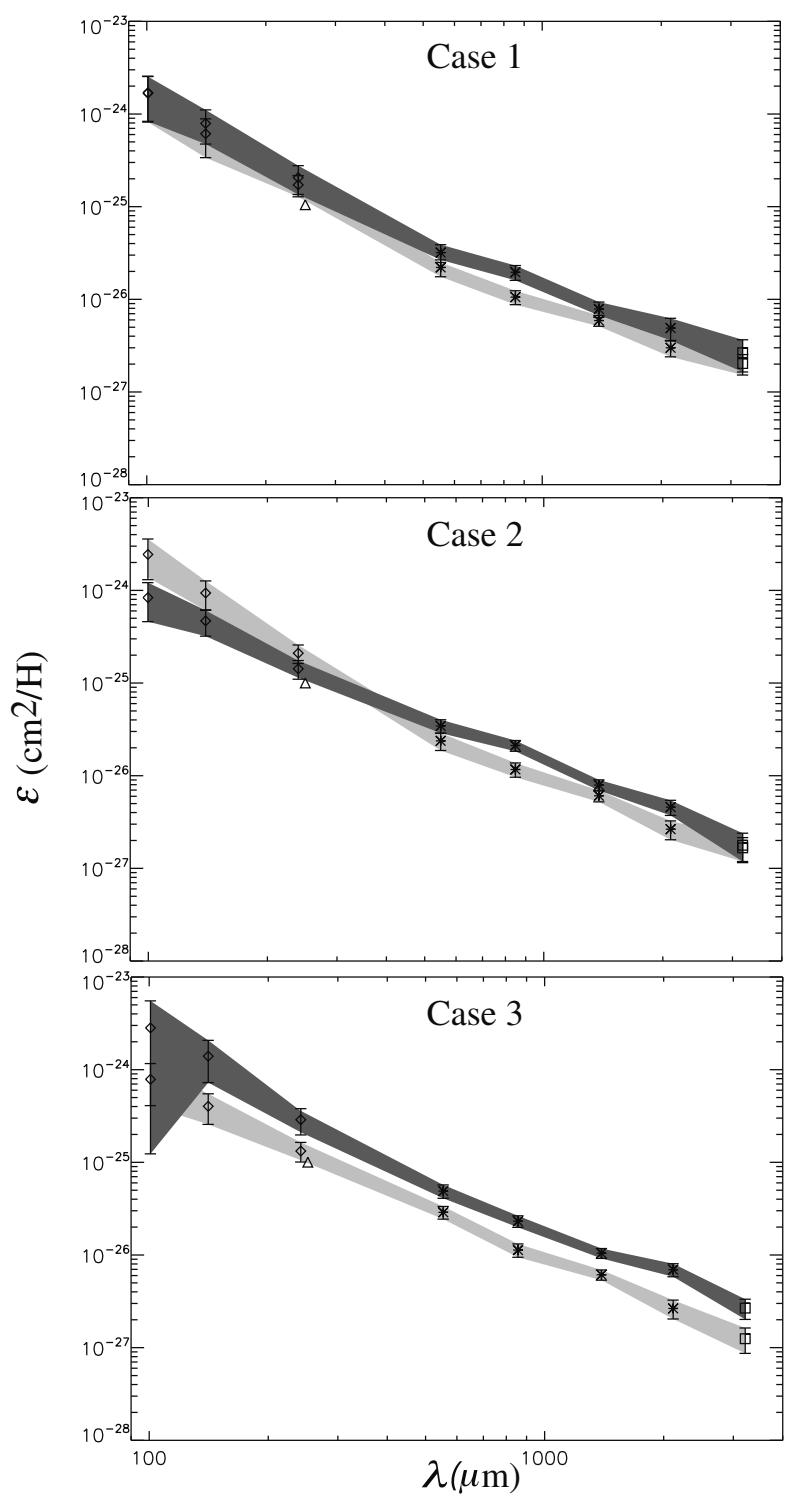

Fig. 3. Median dust emissivity SEDs for case 1 (regular: upper panel), case 2 (colder CO: middle panel) and case 3 (warmer CO: lower panel). The DIRBE, Archeops and WMAP data correspond to diamond, star, and square symbols, respectively. The shaded areas show the $\pm 1-\sigma$ dispersion around each emissivity SED, in dark grey for the atomic phase and in light grey for the molecular phase. The triangle symbol at $\lambda=250 \mu \mathrm{m}$ corresponds to the emissivity value derived by Boulanger et al. (1996) for the diffuse atomic medium at $250 \mu \mathrm{m}$.

produce a wavelength-independent difference between the two emissivity SEDs. Looking at the median values of the emissivity ratios in the two phases presented in Table 4, we can see that the emissivity in the atomic phase is higher that in the molecular phases at all wavelengths, by about $30 \%$ for the assumed $X_{\mathrm{CO}}$. This difference could be accounted for by a change in the $X_{\mathrm{CO}}$ value, which would then have to be slightly lower than the one assumed, $\mathrm{X}_{\mathrm{CO}}=1.6 \times 10^{20} \mathrm{H}_{2} / \mathrm{cm}^{2} /(\mathrm{Kkm} / \mathrm{s})$.

\subsection{Case 2: colder CO}

This case includes regions where dust in the molecular phase is significantly colder than in the surrounding atomic medium. It is of the greatest interest when comparing our results with those obtained using the PRONAOS data in the Taurus and the 
Table 1. Median values of the dust emissivity in the molecular and the atomic phase for each studied case: case 1 (regular), case 2 (colder CO), case 3 (warmer $\mathrm{CO}$ ).

\begin{tabular}{llcccccc}
\hline \hline \multicolumn{2}{c}{ Instrument $\lambda$} & \multicolumn{2}{c}{ Case 1: regular } & \multicolumn{2}{c}{ Case 2: colder CO } & \multicolumn{2}{c}{ Case 3: warmer CO } \\
& $(\mu \mathrm{m})$ & $\epsilon_{\mathrm{HI}}$ & $\epsilon_{\mathrm{CO}}$ & $\epsilon_{\mathrm{HI}}$ & $\epsilon_{\mathrm{CO}}$ & $\epsilon_{\mathrm{HI}}$ & $\epsilon_{\mathrm{CO}}$ \\
\hline DIRBE & 100 & $1.7 \pm 0.9 \times 10^{-24}$ & $1.7 \pm 0.9 \times 10^{-24}$ & $8.4 \pm 3.8 \times 10^{-25}$ & $2.4 \pm 1.1 \times 10^{-24}$ & $2.8 \pm 2.7 \times 10^{-24}$ & $7.8 \pm 3.8 \times 10^{-25}$ \\
DIRBE & 140 & $8.0 \pm 3.2 \times 10^{-25}$ & $6.2 \pm 2.8 \times 10^{-25}$ & $4.7 \pm 1.5 \times 10^{-25}$ & $9.4 \pm 3.3 \times 10^{-25}$ & $1.4 \pm 0.7 \times 10^{-24}$ & $4.0 \pm 1.5 \times 10^{-25}$ \\
DIRBE & 240 & $2.1 \pm 0.7 \times 10^{-25}$ & $1.8 \pm 0.5 \times 10^{-25}$ & $1.4 \pm 0.3 \times 10^{-25}$ & $2.1 \pm 0.5 \times 10^{-25}$ & $2.9 \pm 0.9 \times 10^{-25}$ & $1.3 \pm 0.3 \times 10^{-25}$ \\
Archeops & 550 & $3.3 \pm 0.7 \times 10^{-26}$ & $2.2 \pm 0.5 \times 10^{-26}$ & $3.4 \pm 0.6 \times 10^{-26}$ & $2.4 \pm 0.5 \times 10^{-26}$ & $4.9 \pm 0.8 \times 10^{-26}$ & $2.9 \pm 0.5 \times 10^{-26}$ \\
Archeops & 850 & $2.0 \pm 0.4 \times 10^{-26}$ & $1.1 \pm 0.2 \times 10^{-26}$ & $2.1 \pm 0.3 \times 10^{-26}$ & $1.2 \pm 0.2 \times 10^{-26}$ & $2.3 \pm 0.3 \times 10^{-26}$ & $1.1 \pm 0.2 \times 10^{-26}$ \\
Archeops & 1400 & $8.0 \pm 1.5 \times 10^{-27}$ & $6.0 \pm 0.8 \times 10^{-27}$ & $7.9 \pm 1.1 \times 10^{-27}$ & $6.1 \pm 0.9 \times 10^{-27}$ & $1.0 \pm 0.1 \times 10^{-26}$ & $6.1 \pm 0.8 \times 10^{-27}$ \\
Archeops & 2100 & $5.0 \pm 1.4 \times 10^{-27}$ & $3.1 \pm 0.6 \times 10^{-27}$ & $4.6 \pm 0.9 \times 10^{-27}$ & $2.6 \pm 0.6 \times 10^{-27}$ & $6.9 \pm 1.1 \times 10^{-27}$ & $2.6 \pm 0.6 \times 10^{-27}$ \\
WMAP & 3200 & $2.7 \pm 1.0 \times 10^{-27}$ & $2.1 \pm 0.5 \times 10^{-27}$ & $1.8 \pm 0.6 \times 10^{-27}$ & $1.7 \pm 0.5 \times 10^{-27}$ & $2.7 \pm 0.7 \times 10^{-27}$ & $1.2 \pm 0.4 \times 10^{-27}$ \\
\hline
\end{tabular}

Error bars represent the $\pm 1-\sigma$ uncertainty estimates.

Table 2. Median values of the emissivity spectral index $(\beta)$ in the molecular and the atomic phase for each studied case and each wavelength.

\begin{tabular}{ccccccc}
\hline \hline$\lambda$ & \multicolumn{2}{c}{ Case 1: regular } & \multicolumn{2}{c}{ Case 2: colder CO } & \multicolumn{2}{c}{ Case 3: warmer CO } \\
$(\mu \mathrm{m})$ & $\beta_{\mathrm{HI}}$ & $\beta_{\mathrm{CO}}$ & $\beta_{\mathrm{HI}}$ & $\beta_{\mathrm{CO}}$ & $\beta_{\mathrm{HI}}$ & $\beta_{\mathrm{CO}}$ \\
\hline 100 & $2.3 \pm 1.7$ & $3.0 \pm 2.3$ & $1.7 \pm 1.9$ & $2.9 \pm 2.1$ & $2.1 \pm 3.3$ & $2.0 \pm 1.7$ \\
140 & $2.4 \pm 0.5$ & $2.5 \pm 0.6$ & $2.1 \pm 0.6$ & $2.8 \pm 0.6$ & $2.8 \pm 0.9$ & $2.1 \pm 0.5$ \\
240 & $2.3 \pm 0.3$ & $2.5 \pm 0.3$ & $1.8 \pm 0.2$ & $2.7 \pm 0.3$ & $2.3 \pm 0.3$ & $1.9 \pm 0.2$ \\
550 & $1.9 \pm 0.3$ & $2.1 \pm 0.2$ & $1.5 \pm 0.2$ & $2.3 \pm 0.2$ & $2.0 \pm 0.3$ & $2.0 \pm 0.3$ \\
850 & $1.6 \pm 0.3$ & $1.3 \pm 0.2$ & $1.5 \pm 0.2$ & $1.5 \pm 0.2$ & $1.7 \pm 0.2$ & $1.6 \pm 0.2$ \\
1400 & $1.5 \pm 0.4$ & $1.3 \pm 0.2$ & $1.6 \pm 0.3$ & $1.5 \pm 0.3$ & $1.3 \pm 0.2$ & $1.5 \pm 0.3$ \\
2100 & $1.2 \pm 0.2$ & $1.3 \pm 0.2$ & $1.7 \pm 0.3$ & $1.6 \pm 0.3$ & $1.3 \pm 0.3$ & $1.7 \pm 0.3$ \\
3200 & $1.2 \pm 0.6$ & $0.8 \pm 0.5$ & $2.1 \pm 0.7$ & $1.3 \pm 0.8$ & $2.2 \pm 0.6$ & $1.4 \pm 0.8$ \\
\hline
\end{tabular}

Error bars represent the $1-\sigma$ uncertainty estimates.

Table 3. Median values of the emissivity spectral index $(\beta)$ for the FIR and submm domain, for each studied cases.

\begin{tabular}{ccccc}
\hline \hline$\lambda$ Range & Phase & Case 1: regular & Case 2: colder CO & Case 3: warmer CO \\
\hline$\beta[100-240] \mu \mathrm{m}$ & $\mathrm{HI}$ & $2.4 \pm 0.5$ & $2.1 \pm 0.6$ & $2.8 \pm 0.9$ \\
& $\mathrm{CO}$ & $2.5 \pm 0.6$ & $2.8 \pm 0.6$ & $2.1 \pm 0.5$ \\
$\beta_{\mathrm{CO}} / \beta_{\mathrm{HI}}$ & & $1.0 \pm 0.5$ & $1.3 \pm 0.5$ & $0.7 \pm 0.6$ \\
$\beta[550-2100] \mu \mathrm{m}$ & $\mathrm{HI}$ & $1.5 \pm 0.2$ & $1.5 \pm 0.2$ & $1.5 \pm 0.1$ \\
& $\mathrm{CO}$ & $1.4 \pm 0.2$ & $1.5 \pm 0.2$ & $1.7 \pm 0.2$ \\
$\beta_{\mathrm{CO}} / \beta_{\mathrm{HI}}$ & & $0.9 \pm 0.3$ & $1.0 \pm 0.2$ & $1.1 \pm 0.2$ \\
\hline
\end{tabular}

Errors bars represent the $1-\sigma$ uncertainty estimates.

Polaris regions, which lead previous authors to propose the presence of fractal dust aggregates in the molecular phase. Our results (see Fig. 3) indicate that the emissivity SED of dust associated with the atomic and molecular medium differ. In the FIR (DIRBE wavelength range), the emissivity spectral index in the molecular phase is significantly higher than in the atomic phase. According to Table 3 the molecular to atomic emissivity ratio $\left(\frac{\beta_{\mathrm{CO}}}{\beta_{\mathrm{HI}}}\right)$ is equal to 1.3 in the FIR domain. However, in the submillimeter (Archeops wavelength range), the emissivity spectra in the two phases become parallel again, and have quite comparable emissivity values for the assumed value of $X_{\mathrm{CO}}$. Matching exactly the submm emissivities in the two phases in the submillimeter range would lead to $X_{\mathrm{CO}}=1.5 \times 10^{20} \mathrm{H}_{2} / \mathrm{cm}^{2} /(\mathrm{Kkm} / \mathrm{s})$. According to Table $3, \frac{\beta_{\mathrm{CO}}}{\beta_{\mathrm{HI}}}=1.0$, indicating that the slope of the submm emissivity is not very different from case 1 , whose ratio is 0.9 . For the adopted $X_{\mathrm{CO}}$, we observe that the emissivity in the molecular phase is higher than that in the atomic phase in the FIR range, by a factor ranging from 3.1 at $100 \mu \mathrm{m}$, to about 1.5 at $240 \mu \mathrm{m}$ (see Table 4$)$. The absolute emissivity values for the two phases tend to become progressively similar around $550 \mu \mathrm{m}$. This behavior cannot be attributed to the assumed $X_{\mathrm{CO}}$ value, which would affect emissivity values at all wavelengths. We also considered the possibility that this could be due to an error on the determination of the dust temperature in the molecular phase. Obtaining the same emissivity slope in the FIR in the two phases would require an increase of the temperature in the molecular phase of 2-3 K, which is larger than the temperature uncertainty, which are between $0.25 \mathrm{~K}$ and $0.72 \mathrm{~K}$. In our analysis, we have so far used the major simplification of a single dust temperature along the line of sight. In Sect. 7, we investigate the effect of this hypothesis on the results. In particular, we consider the effect of the temperature mixing due to the grain size distribution, the LOS mixing of the radiation field and the grain composition.

\subsection{Case 3: warmer CO}

In this case, the dust temperature in the molecular phase is higher than the dust temperature in the atomic phase. Taking into account the error bars, the behavior of the emissivity spectrum is qualitatively the same as in case 1 . For the assumed $X_{\mathrm{CO}}$, we note that the atomic emissivities are significantly higher than the molecular ones at all wavelengths. To reproduce the emissivity in the submm, we would require 
Table 4. Median values of the ratio between the dust emissivity in the molecular phase and in the atomic phase for each studied case. Error bars represent the $\pm 1-\sigma$ dispersion around each value.

\begin{tabular}{cccc}
\hline \hline$\lambda(\mu \mathrm{m})$ & $\begin{array}{c}\epsilon_{\mathrm{CO}} / \epsilon_{\mathrm{HI}} \\
\text { Case 1: regular }\end{array}$ & $\begin{array}{c}\epsilon_{\mathrm{CO}} / \epsilon_{\mathrm{HI}} \\
\text { Case 2: colder CO }\end{array}$ & $\begin{array}{c}\epsilon_{\mathrm{CO}} / \epsilon_{\mathrm{HI}} \\
\text { Case 3: warmer CO }\end{array}$ \\
\hline 100 & $0.8 \pm 0.7$ & $3.1 \pm 2.9$ & $0.2 \pm 0.2$ \\
140 & $0.7 \pm 0.5$ & $2.1 \pm 1.6$ & $0.2 \pm 0.2$ \\
240 & $0.8 \pm 0.4$ & $1.5 \pm 0.8$ & $0.4 \pm 0.2$ \\
550 & $0.8 \pm 0.3$ & $0.7 \pm 0.2$ & $0.6 \pm 0.2$ \\
850 & $0.6 \pm 0.2$ & $0.5 \pm 0.2$ & $0.5 \pm 0.2$ \\
1400 & $0.8 \pm 0.2$ & $0.8 \pm 0.2$ & $0.5 \pm 0.1$ \\
2100 & $0.7 \pm 0.2$ & $0.6 \pm 0.3$ & $0.3 \pm 0.2$ \\
3200 & $0.7 \pm 0.2$ & $0.7 \pm 0.4$ & $0.3 \pm 0.3$ \\
\hline
\end{tabular}

$X_{\mathrm{CO}}=1.1 \times 10^{20} \mathrm{H}_{2} / \mathrm{cm}^{2} /(\mathrm{Kkm} / \mathrm{s})$. The results of this case show that when the dust temperature in the atomic phase is close to or colder than the molecular phase, grains emission properties seem to be the same.

\section{Possible biases caused by assumptions}

In the study presented above, we assumed a single dust temperature along each LOS. This is probably overly simplistic, since we expect a mixture of temperatures for various reasons. First, dust exhibits a size distribution, and dust temperatures depend upon grain size for a given radiation field. Second, the ISRF strength probably varies along any given LOS within the Galactic plane, even towards the external Galactic regions studied here. Third, composition variations in the dust could produce temperature variations, which could also affect our results. Since we divide the observed sky brightness by the black-body function at a single temperature, slight systematic biases in the dust temperature caused by these effects could in principle explain the inferred variations of the dust emissivity, particularly in the FIR region, where the black-body function is non-linear with respect to the dust temperature.

In this section, we explore those 3 possibilities and test the robustness of our results to temperature variations along the LOS induced by either grain size distribution, ISRF strength mixing or grain composition variations. In all cases, we produce predicted emission SEDs using a pertinent dust emission model, which includes the additional complexity, apply the same treatment applied to the true data to the modelled SEDs, and compare the derived emissivity curves with that of the model.

\subsection{Effect of the grain size distribution}

To test the influence of temperature caused by the BG size distribution, we generated model SEDs at various ISRF intensities ( $X_{\text {ISRF }}$ ), using the dust model of Désert et al. (1990), which includes a realistic size distribution for large grains and computes the induced temperature mixing, by taking into account the effects of grain temperature fluctuations. Model SEDs were computed in all instrument filters used in our study, using the proper color correction to match the flux convention of each instrument. We then applied the same processing to the model SEDs as applied to the actual data. As the model assumes a dust emissivity with $\beta=2$ over the entire wavelength range considered here, we expect in that case to recover a simple power law with slope $\beta=2$. We performed the test over $X_{\mathrm{ISRF}}$ values ranging from 0.05 to 10 times the solar neighbourhood ISRF strength. Ratios of the predicted emissivity values inferred from

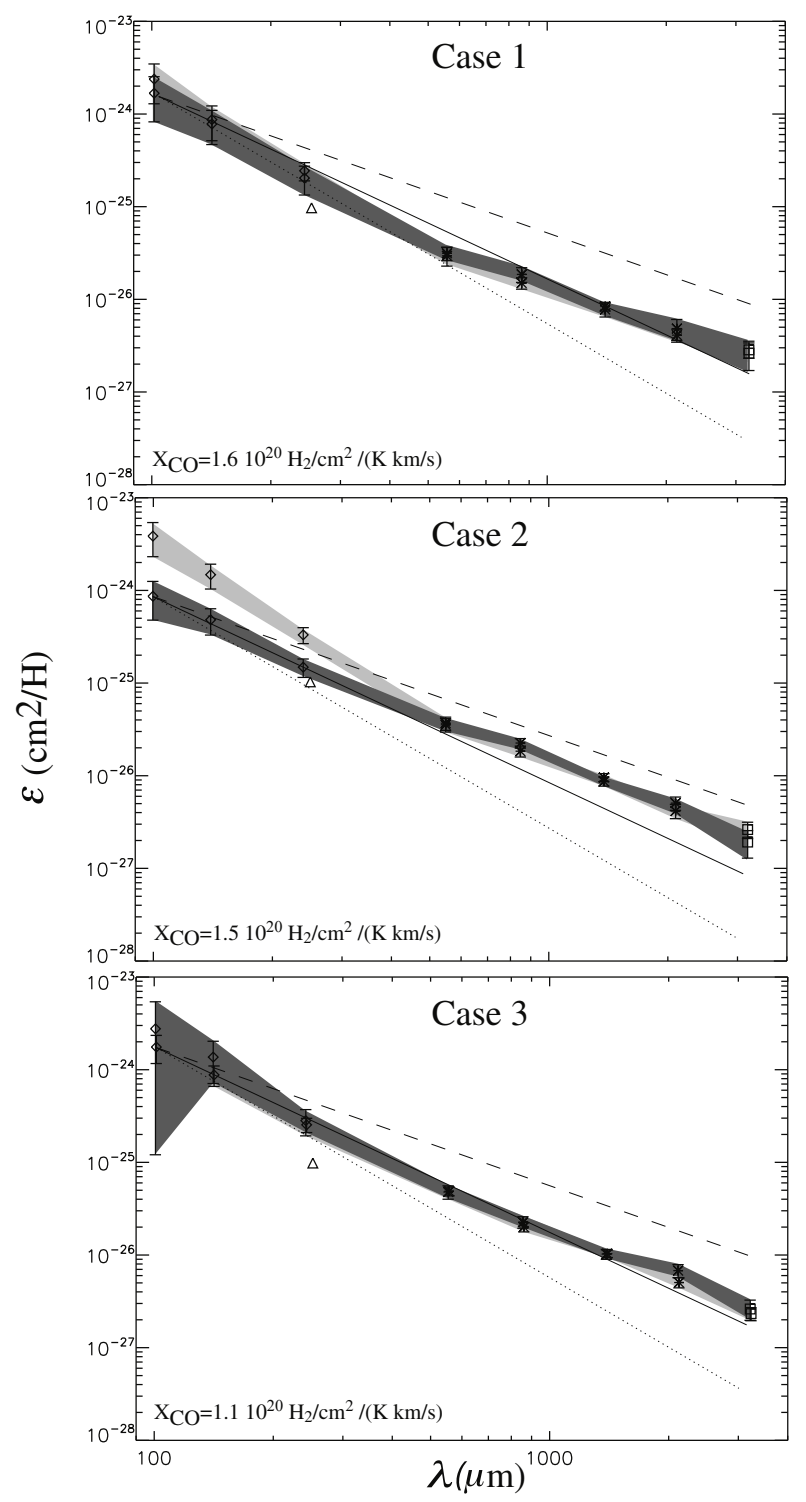

Fig. 4. Same as Fig. 3 but with the molecular emissivity scaled to match that of the atomic phase in the range $550 \mu \mathrm{m}<\lambda<3 \mathrm{~mm}$. The corresponding $X_{\mathrm{CO}}$ values are given in the figure. For comparison, power law spectra with $\beta=1.5$ (dashed), $\beta=2.0$ (solid) and $\beta=2.5$ (dot) are shown, normalised to the atomic emissivity at $100 \mu \mathrm{m}$.

the model and the apparent emissivity obtained with our procedure are given in Table 5, normalised to the emissivity value in the WMAP band. Figure 5 is a graphical representation of Table 5. The left panel shows that the emissivity ratio is close to 1 . We therefore recover emissivity spectra very similar to the input power law, indicating that no significant bias is introduced by not considering the LOS temperature mixing induced by a realistic dust size distribution. We also checked the influence of changing the spectral shape of the ISRF, in particular increasing the UV content as expected close to young stars. This test showed that the shape of the ISRF does not affect our results, because BGs absorb uniformly across the entire visible to ultraviolet range of the model used. We conclude that the method described above introduces no significant bias in that case. 
Table 5. Ratios of the predicted to apparent emissivities, using the same processing as for the data with different models.

\begin{tabular}{cccccccccc}
\hline \hline$\lambda(\mu \mathrm{m})$ & \multicolumn{3}{c}{ Grain size distribution } & \multicolumn{3}{c}{ ISRF strength mixture } & \multicolumn{3}{c}{ Grain composition } \\
& $X_{\mathrm{ISRF}}=0.05$ & $X_{\mathrm{ISRF}}=0.5$ & $X_{\mathrm{ISRF}}=4$ & $\alpha=2.5$ & $\alpha=2$ & $\alpha=1.25$ & $T_{2}=10 \mathrm{~K}$ & $T_{2}=15 \mathrm{~K}$ & $T_{2}=22 \mathrm{~K}$ \\
\hline 100 & 1.13 & 0.89 & 0.93 & 1.20 & 1.46 & 3.06 & 1.06 & 1.29 & 1.16 \\
140 & 1.13 & 0.96 & 0.98 & 1.23 & 1.45 & 2.66 & 1.12 & 1.27 & 1.14 \\
240 & 1.11 & 1.00 & 1.02 & 1.17 & 1.29 & 1.84 & 1.03 & 1.19 & 1.13 \\
550 & 1.07 & 1.03 & 1.03 & 1.09 & 1.12 & 1.27 & 0.96 & 1.05 & 1.04 \\
850 & 1.04 & 1.02 & 1.02 & 1.05 & 1.07 & 1.14 & 1.07 & 1.10 & 1.08 \\
1400 & 1.00 & 0.99 & 0.99 & 1.01 & 1.01 & 1.05 & 1.00 & 1.04 & 1.05 \\
2100 & 0.99 & 0.99 & 0.99 & 0.99 & 1.00 & 1.01 & 0.98 & 1.01 & 1.03 \\
\hline
\end{tabular}

Columns 2-4: using the Désert et al. (1990) model at various ISRF strength $\left(X_{\text {ISRF }}\right)$. Columns 5-7: assuming a mixture of the radiation field intensity on each LOS, using the model by Dale et al. (2001) for various values of the mixing parameter $\alpha$. Columns 8-10: using the two component model of Finkbeiner et al. (1999) for different temperatures of the warm component $\left(T_{2}\right)$.

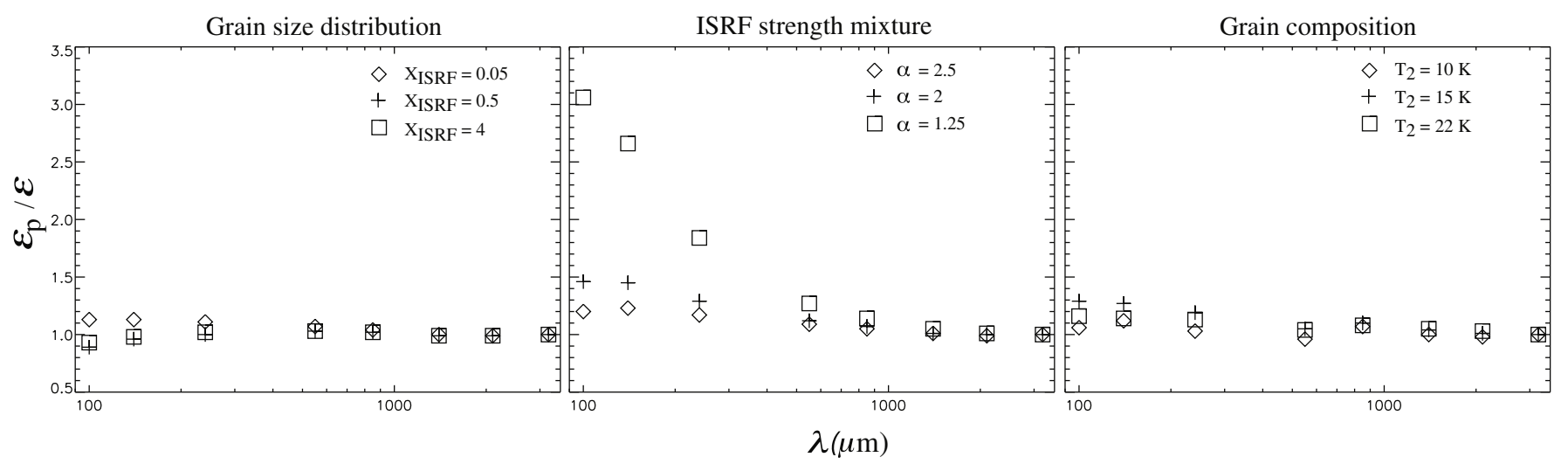

Fig. 5. Ratios of the predicted $\left(\epsilon_{\mathrm{p}}\right)$ to apparent emissivities $(\epsilon)$, using the same processing as for the data with different modelled SEDs. Left panel: using the Désert et al. (1990) model at various ISRF strengths $\left(X_{\mathrm{ISRF}}\right)$. Middle panel: assuming a mixture of the radiation field intensity on each line of sight, using the model description proposed by Dale et al. (2001) for various values of the mixing parameter $\alpha$. Right panel: using the two component model of Finkbeiner et al. (1999) for different temperatures of the warm component $\left(T_{2}\right)$.

\subsection{Effect of the ISRF strength mixture}

To test the influence of the variations in the ISRF strength mixture along the LOS, we use the model proposed by Dale et al. (2001), who introduced the concept of local SED combination, assuming a power-law distribution of dust mass subjected to a given heating intensity $\mathrm{d} M_{\mathrm{d}}\left(X_{\mathrm{ISRF}}\right)$, to interpret the emission by external galaxies:

$\mathrm{d} M_{\mathrm{d}}\left(X_{\mathrm{ISRF}}\right) \propto X_{\mathrm{ISRF}}^{-\alpha} \mathrm{d} X_{\mathrm{ISRF}}$,

where $\alpha$ controls the relative contribution of the various ISRF intensities $\left(X_{\text {ISRF }}\right)$ to the SEDs. Dale et al. (2001) used ISRF strengths values in the range $0.3<X_{\text {ISRF }}<10^{5}$ and derived $\alpha$ values in the range $1<\alpha<2.5$ for external galaxies. We first computed emission spectra using the Désert et al. (1990) model at various $X_{\mathrm{ISRF}}$ values (noted $I_{v}^{\bmod }\left(X_{\mathrm{ISRF}}\right)$ ), then summed them over the same $X_{\text {ISRF }}$ range as proposed by Dale et al. (2001), according to

$I_{v}^{\mathrm{tot}}=\frac{\sum_{X_{\mathrm{ISRF}}} I_{v}^{\mathrm{mod}}\left(X_{\mathrm{ISRF}}\right) \times X_{\mathrm{ISRF}}^{-\alpha}}{\sum_{X_{\mathrm{ISRF}}} X_{\mathrm{ISRF}}^{-\alpha}}$.

In Fig. 5 (middle plot), it can be seen that significant departures from model predictions can occur in the FIR, close to the peak of the emission, for flat $X_{\text {ISRF }}$ distributions (i.e., low $\alpha$ values). These deviations become progressively smaller towards steeper distribution, as expected. We note that the apparent dust temperature derived from the fit decreases as $\alpha$ increases, which can be used to constrain $\alpha$ values. From the model predictions, we corrected our emissivity estimates, using the apparent dust temperature for each measured SED to infer the true $\alpha$ value and then deduce the factor we have to apply to correct our values, knowing the ratio of the predicted to apparent emissivity. In general, assuming a single dust temperature along the LOS causes the emissivity values between 100 and $550 \mu \mathrm{m}$ to be underestimated. For most of the regions considered here, the apparent temperature is moderate, which is indicative of steep $X_{\mathrm{ISRF}}$ mixing with $\alpha$ close to 2.5 .

The resulting corrected median emissivity values are shown in Fig. 6, after renormalisation by $X_{\mathrm{CO}}$. Comparison with Fig. 4 shows that $X_{\text {ISRF }}$ mixing has little impact on our results. In particular, both the break in the emissivity slope near $500 \mu \mathrm{m}$ and the dust emissivity increase in the molecular phase in case 2 are still present. We note that the amplitude of the emissivity increase is slightly lower after the correction, as shown in Table 6, with a median ratio of $2.0 \pm 1.6$ between the 2 spectra at $100 \mu \mathrm{m}$ after correction, instead of 3.1 , before renormalisation by $X_{\mathrm{CO}}$. The emissivity slopes in the FIR are equal to $2.7 \pm 0.6$ and $2.3 \pm 0.6$, in the FIR for the molecular and atomic phase, respectively, and $1.5 \pm 0.2$ and $1.6 \pm 0.2$ in the submm.

\subsection{Effect of the grain composition}

We use the Finkbeiner et al. (1999) model to test the influence of dust composition on our results. In the framework of this model, the shape of the submm emission is assumed to be caused by a mixture of silicate and graphite grains that reach different temperatures $\left(T_{1}\right.$ and $T_{2}$ for the silicate and graphite components, 
Table 6. Median values of the dust emissivity in the molecular and the atomic phase for each studied case, after correction of a mixture of the radiation field intensity along the line of sight.

\begin{tabular}{ccccccc}
\hline \hline$\lambda$ & \multicolumn{2}{c}{ Case 1: regular } & \multicolumn{2}{c}{ Case 2: colder CO } & \multicolumn{2}{c}{ Case 3: warmer CO } \\
$(\mu \mathrm{m})$ & $\epsilon_{\mathrm{HI}}$ & $\epsilon_{\mathrm{CO}}$ & $\epsilon_{\mathrm{HI}}$ & $\epsilon_{\mathrm{CO}}$ & $\epsilon_{\mathrm{HI}}$ & $\epsilon_{\mathrm{CO}}$ \\
\hline 100 & $1.8 \pm 0.9 \times 10^{-24}$ & $1.6 \pm 0.7 \times 10^{-24}$ & $1.2 \pm 0.6 \times 10^{-24}$ & $2.0 \pm 0.9 \times 10^{-24}$ & $2.7 \pm 2.5 \times 10^{-24}$ & $8.5 \pm 4.5 \times 10^{-25}$ \\
140 & $8.7 \pm 3.3 \times 10^{-25}$ & $7.5 \pm 2.6 \times 10^{-25}$ & $6.1 \pm 2.6 \times 10^{-25}$ & $8.2 \pm 2.8 \times 10^{-25}$ & $1.3 \pm 0.7 \times 10^{-24}$ & $4.6 \pm 1.8 \times 10^{-25}$ \\
240 & $2.3 \pm 0.8 \times 10^{-25}$ & $1.9 \pm 0.4 \times 10^{-25}$ & $1.8 \pm 0.5 \times 10^{-25}$ & $1.9 \pm 0.4 \times 10^{-25}$ & $2.8 \pm 0.8 \times 10^{-25}$ & $1.5 \pm 0.3 \times 10^{-25}$ \\
550 & $3.3 \pm 0.8 \times 10^{-26}$ & $2.4 \pm 0.5 \times 10^{-26}$ & $3.9 \pm 0.6 \times 10^{-26}$ & $2.3 \pm 0.5 \times 10^{-26}$ & $5.1 \pm 0.8 \times 10^{-26}$ & $3.0 \pm 0.5 \times 10^{-26}$ \\
850 & $2.0 \pm 0.4 \times 10^{-26}$ & $1.1 \pm 0.1 \times 10^{-26}$ & $2.3 \pm 0.3 \times 10^{-26}$ & $1.2 \pm 0.2 \times 10^{-26}$ & $2.4 \pm 0.3 \times 10^{-26}$ & $1.2 \pm 0.2 \times 10^{-26}$ \\
1400 & $7.9 \pm 1.5 \times 10^{-27}$ & $5.9 \pm 0.8 \times 10^{-27}$ & $8.1 \pm 1.1 \times 10^{-27}$ & $5.9 \pm 0.8 \times 10^{-27}$ & $1.0 \pm 0.1 \times 10^{-26}$ & $6.0 \pm 0.8 \times 10^{-27}$ \\
2100 & $5.0 \pm 1.4 \times 10^{-27}$ & $3.0 \pm 0.6 \times 10^{-27}$ & $4.6 \pm 0.9 \times 10^{-27}$ & $2.6 \pm 0.6 \times 10^{-27}$ & $6.8 \pm 1.1 \times 10^{-27}$ & $2.6 \pm 0.6 \times 10^{-27}$ \\
3200 & $2.7 \pm 1.0 \times 10^{-27}$ & $2.0 \pm 0.5 \times 10^{-27}$ & $1.8 \pm 0.6 \times 10^{-27}$ & $1.7 \pm 0.5 \times 10^{-27}$ & $2.7 \pm 0.7 \times 10^{-27}$ & $1.2 \pm 0.4 \times 10^{-27}$ \\
\hline
\end{tabular}

Error bars represent the $\pm 1-\sigma$ uncertainty estimates.

respectively), the temperatures being linked by the radiation field. In principle, this could also affect our interpretation, since we assumed a single dust temperature to derive the dust emissivity spectrum. Unlike Finkbeiner et al. (1999) however, we used the same $\lambda^{-2}$ power law emissivity for each grain component, to simplify the comparison between the recovered emissivity and the model. We computed the emissivity ratio at various temperatures of the graphitic component ranging from $8 \mathrm{~K}$ to $22 \mathrm{~K}$ (see the right plot of Fig. 5). Table 5 shows that in this case the bias introduced by our simplification hypothesis does not significantly impact our results. Similar tests using various emissivity power laws for the two components gave equally good results.

\section{Discussion}

Regarding the absolute values of the derived emissivities, it can be seen in Fig. 3 that the values we derived for the atomic phase are in rough agreement with that derived by Boulanger et al. (1996) $\left(10^{-25} \mathrm{~cm}^{2} / \mathrm{H}\right.$ at $\left.250 \mu \mathrm{m}\right)$. This agreement indicates that the regions we have selected in this study, which are mainly located towards the Galactic 2nd quadrant, have similar dust content and properties to the solar neighbourhood. A closer inspection of Fig. 3 shows that our emissivity values in the atomic phase are systematically higher than the solar neighbourhood values by a factor of $1.9,1.3$, and 2.6 for cases 1,2 , and 3 , respectively. The corresponding median dust temperatures derived are $15.0 \mathrm{~K}, 16.9 \mathrm{~K}$, and $14.0 \mathrm{~K}$ respectively (see Table 7), which are also lower than the temperature inferred by Boulanger et al. (1996) for the diffuse ISM in the solar neighbourhood (17.5 K).

We also emphasize that the atomic and molecular emissivity spectra for cases 1 and 3 could be reproduced over the full wavelength range studied by slightly decreasing the $X_{\mathrm{CO}}$ value used for the column density conversion. We therefore consider it likely that the actual $X_{\mathrm{CO}}$ value is slightly lower than the assumed value of $2.3 \times 10^{20} \mathrm{H}_{2} / \mathrm{cm}^{2} /(\mathrm{K} \mathrm{km} / \mathrm{s})$ in those cases. In case 2 , however, the emissivity spectra have a significantly different shape and cannot be reproduced over the full wavelength range by simply changing the $X_{\mathrm{CO}}$ value. We note however that a similar decrease to that in case 1 would improve the match in the submm and millimeter range.

Regarding the spectral shape of the emissivity, the results shown in Sect. 6.2 indicate that the FIR dust emissivity of dust associated with molecular regions is significantly higher than that of the surrounding atomic medium, in cases where the dust temperature is colder in the molecular phase than in the atomic phase (Case 2). Taking into account the error bars derived in Sect. 5.1 and shown in Fig. 4, this result is significant at the $5-\sigma$ level. This result is in qualitative agreement with the result obtained by Stepnik et al. (2003), who showed that an increase in the dust emissivity by a factor of 3-4 in the molecular phase is required to explain the low dust temperature in that phase. We note that their study was limited to the wavelength range from 200 to $600 \mu \mathrm{m}$. The increase that we found for the DIRBE wavelength range is therefore of the same order, although slightly smaller, than that derived by Stepnik et al. (2003) for a particular cold molecular filament in Taurus. In our case 2 regions, we note that on average the dust is $3.2 \mathrm{~K}$ colder in the molecular phase than in the atomic phase. This is also similar to, although slightly lower than, the difference observed by Stepnik et al. (2003) in the Taurus filament (4.8 K). Our result therefore confirms the hypothesis proposed by Bernard et al. (1999) and Stepnik et al. (2003) that dust aggregation may be responsible for the decrease in the dust equilibrium temperature. In quiescent molecular clouds of case 2, aggregation of BGs in fractal clusters is a likely cause of the FIR emissivity increase, the corresponding decrease in the dust equilibrium temperature being a consequence of the higher emissivity and more efficient cooling.

Stepnik et al. (2003) inferred an increase in emissivity by a constant factor with wavelength. Their data did not allow them to measure directly the spectral shape of the emissivity, and they used an average $\beta$ index over the FIR wavelength range of the PRONAOS experiment. Their approximation of a wavelength independent increase in the emissivity was supported by the results of discrete dipole approximation calculations for dust aggregates (e.g., Bazell \& Dwek 1990) that predict a constant absorptivity increase for fractal aggregates all the way into the millimeter. Our results however show that the emissivity spectrum in the molecular phase follows the same power law as in the atomic phase. Although the dust emissivity in the cold molecular phase is significantly higher in the FIR, it recovers the atomic phase values in the submm and $\mathrm{mm}$ ranges.

We note however that reproducing the temperature difference by increasing the dust emissivity only requires the emissivity to be higher in the FIR, where large grains emit most of their energy. Therefore, the close agreement between the emissivities derived for the submm and $\mathrm{mm}$ does not therefore the thermal argument upon which the conclusion of dust aggregates is based. A simple calculation of the thermal equilibrium shows that the measured emissivity increase in case 2 would produce a temperature decrease of about $2 \mathrm{~K}$, assuming that the increase below $\lambda=100 \mu \mathrm{m}$ equals that at $100 \mu \mathrm{m}$. This is slightly lower than the observed temperature decrease, which could indicate that the emissivity continues increasing below $100 \mu \mathrm{m}$.

In all cases studied, the emissivity spectra derived are steeper in the FIR, with spectral index of $\beta \simeq 2.4$ and flatten in the submm and $\mathrm{mm}$ regime, where the spectral index reaches 




Fig. 6. Median dust emissivity SEDs for the 3 cases (see Fig. 3 for the description of the curves) corrected for the hypothesis of a mixture of different interstellar radiation field along the line of sight. The molecular emissivity has been scaled to match that of the atomic phase in the range $550 \mu \mathrm{m}<\lambda<3 \mathrm{~mm}$. The triangle corresponds to the emissivity value derived by Boulanger et al. (1996) in the diffuse medium.

$\beta \simeq$ 1.5. Taking into account, the error bars derived in Sect. 5.1 and shown in Fig. 4, this result is significant at the $10-\sigma$ and $15-\sigma$ level for cases 1 and 2 , respectively. We note that attributing the flattening to calibration errors would require the calibration of both the Archeops and WMAP experiments to be wrong by more than a factor of 2 in the same direction, which is very unlikely. This behavior could in principle be due to the presence of two dust components at different equilibrium temperatures, as proposed in phenomenological models with two temperature components (e.g., Finkbeiner et al. 1999). Such models have been invoked to explain the flatness of submm emission spectrum, but require the existence of a cold component with $T_{\mathrm{d}} \simeq 9 \mathrm{~K}$, whose origin in the diffuse ISM remains to be understood. However, we note that the spectral indices that we obtained in this work are in good agreement with those proposed in the Finkbeiner et al. (1999) model.
A more physical approach consists of interpreting the submm flattening by intrinsic dust properties. To explain the FIR/mm dust emission, Mény et al. (2007) proposed a model based on some specific properties of the amorphous state. First, they considered a temperature-independent emission caused by excitation of acoustic lattice vibrations, coming from the coupling between the electromagnetic fields and a disordered charge distribution (DCD) that characterizes the amorphous nature of dust. Their description also takes into account resonant absorption and relaxation processes associated with localized asymmetric two-level systems (TLS) in the grains, which produce additional emission with emissivity that depends on both temperature and wavelength. Within this model, the emissivity spectral index is therefore predicted to change with both wavelength and temperature. The behaviour of a flattening of the spectrum in the millimeter range is in qualitative agreement with their model, although the data do not allow us to test further a possible dependence with dust temperature, since the temperature range that we can sample here is limited. Finally, we note that in this model, the TLS phenomenon responsible for the emission above $\lambda \simeq 600 \mu \mathrm{m}$ is of a different nature than the vibration of DCD in the amorphous lattice producing the FIR emission. It is therefore possible that fractal aggregates consisting of amorphous individual grains also exhibit a change in properties between the FIR and the submm. In particular, the TLS phenomenon operating at atomic scale may be less sensitive to the dust grains being gathered into aggregates, while the DCD vibration, which is a global phenomenon, is expected to exhibit an excess emission, as predicted by classical DDA calculations. This may offer a possible physical interpretation to the emissivity increase of dust in the molecular phase as being limited to the FIR region. Obviously, detailed modelling of the interaction between the electromagnetic wave and a fractal aggregate consisting of amorphous material is needed to further investigate this issue.

\section{Conclusions}

We have analysed the dust emission from the outer Galactic plane region using DIRBE, Archeops, and WMAP data from $100 \mu \mathrm{m}$ to $3.2 \mathrm{~mm}$. We have performed a correlation study of the FIR-mm emission with gas tracers in individual regions, and derived the average equilibrium temperature of large dust grains in both molecular and atomic phases in a set of regions along the Galactic plane. We used this temperature to derive the emissivity spectra for each phase and region. We classified regions into 3 classes, according to the relative temperature of the dust associated with the molecular and atomic phases. For each class, we derived the median emissivity profile.

We showed that the emissivity spectra are always steeper in the FIR $(\lambda<600 \mu \mathrm{m})$ and flatten in the submm and $\mathrm{mm}$. In regions where dust is significantly colder in the molecular phase than in the surrounding atomic medium, we produced an increase in the emissivity by a factor of $\simeq 3$ in the FIR. However, we showed that the emissivity increase is restricted to the FIR range; the emissivity spectra for the dust in the atomic and molecular phases become comparable again in the submm and mm wavelength range.

The observed break in the emissivity spectrum, which appears to be a general phenomenon, is consistent with the phenomenological all-sky fit of the FIRAS data of Finkbeiner et al. (1999). It is also in qualitative agreement with the dust emission model of Mény et al. (2007), which invokes quantum effects in amorphous solids to explain the flatness of the observed submm 
emission spectrum and also produces a break in the emissivity slope around $600 \mu \mathrm{m}$.

We interpret the FIR emissivity increase in molecular clouds containing cold dust as being caused by the coagulation of large grains into fractal aggregates. Previous observations, obtained over much smaller portions of the sky by Stepnik et al. (2003), showed that dust aggregation could lead to an increase in the dust emissivity by a factor of 3-4, to explain the unusually low dust temperatures observed. The fact that emissivities do reconcile in the submillimeter is not in agreement with DDA calculations of simple aggregates and the physical reason for this remains unclear. We however propose that this may be related to the amorphous nature of the grains contained in the aggregates.

Finally, the absence of a detectable FIR emissivity increase in regions where the dust temperature in the molecular phase is similar or higher than that in the atomic phase is interpreted as the absence of fractal grains in those environments. This is probably because the formation of such aggregates is prevented by the star formation activity, while it is favoured in more quiescent regions where turbulence is milder.

\section{References}

Abergel, A., Boulanger, F., Mizuno, A., et al. 1994, ApJ, 423, L59 Abergel, A., Boulanger, F., Fukui, Y., et al. 1995, A\&AS, 111, 483 Allamandola, L. J., Tielens, A. G. G. M., \& Barker, J. R. 1989, ApJS, 71, 733 Bacmann, A., Lefloch, B., Ceccarelli, C., et al. 2002, A\&A, 389, 6 Bazell, D., \& Dwek, E. 1990, ApJ, 360, 142

Bennett, C., Hill, R., \& Hinshaw, G. 2003, ApJS, 148, 117

Benoit, A., Amblard, A., Ansari, R., et al. 2002, Astropart. Phys., 101 Bernard, J.-Ph., Boulanger, F., Désert, F. X, Puget, J. L. 1992, A\&A, 263, 258

Bernard, J.-Ph., Abergel, A., Ristorcelli, I., et al. 1999, A\&A, 347, 640 Boulanger, F., Prevot, M. L., \& Gry, C. 1994, A\&A, 284, 956

Boulanger, F., Abergel, A., Bernard, J.-Ph., et al. 1996, A\&A, 312, 256

Cambrésy, L. 1999, A\&A, 345, 965

Cambrésy, L., Boulanger, F., Lagache, G., et al. 2001a, A\&A, 375, 999
Cambrésy, L., Reach, W. T., Beichman, T. H., \& Jarret, T. H. 2001b, ApJ, 555, 563

Caselli, P., Walmsley, C. M., Tafalla, M., et al. 1999, ApJ, 523, 165

Dale, D. A., Helou, G., Contursi, A., et al. 2001, ApJ, 549, 215

Dame, T. M., Hartmann, D., \& Thaddeus, P. 2001, ApJ, 547, 792

Delabrouille, J., Cardoso, J.-F., Le Jeune, M., et al. 2009, A\&A, 493, 835

Désert, F.-X., Boulanger, F., \& Puget, J. L. 1990, A\&A, 237, 215

Dickey, J. M., \& Lockman, F. J. 1990, Annu. Rev. Astron. Astrophys., 28, 215

Draine, B. T., \& Anderson, N. 1985, ApJ, 292, 494

Draine, B. T., \& Lazarian, A. 1998, ApJ, 494, L19

Dupac, X., Bernard, J.-Ph., Boudet, N., et al. 2003, A\&A, 404, L11

Finkbeiner, D. P., Davis, M., \& Schlegel, D. J. 1999, ApJ, 524, 867

Fixsen, D. J., Cheng, E. S., Cottingham, D. A., et al. 1994, ApJ, 420, 457

Fixsen, D. J., Weiland, J. L., Brood, S., et al. 1997, ApJ, 490, 482

Hartmann, D. 1994, Ph.D. thesis, The Leiden/Dwingeloo Survey of Galactic Neutral Hydrogen, University of Leiden

Hartmann, D., \& Burton, W. B. 1996, Atlas of Galactic Neutral Hydrogen (Cambridge University Press)

Hinshaw, G., Barnes, C., Bennett, et al. 2003, ApJS, 148, 63

Kemper, F., Vriend, W. J., \& Tielens, A. G. G. M. 2004, ApJ, 609, 826

Lagache, G., Abergel, A., Boulanger, F., \& Puget, J. L. 1998, A\&A, 333, 709

Laureijs, R. J., Clark, F. O., \& Prusti, T. 1991, ApJ, 372, 185

Laureijs, R. J., Fukui, Y., Helou, G., et al. 1995, ApJS, 101, 87

Laureijs, R. J., Haikala, L., Burgdorf, M., et al. 1996, A\&A, 315, L317

Macias-Perez, J. F., Lagache, G., Maffei, B., et al. 2007, A\&A, 467, 1313

Magnani, L., \& Onello, J. S. 1995, ApJ, 443, 169

Mathis, J. S., Mezger, P. G., \& Panagia, N. 1983, A\&A, 128, 212

Mény, C., Gromov, V., Boudet, N., et al. 2007, A\&A, 468, 171

Spitzer, L. 1978, Physical Processes in the interstellar medium (New-York: Wiley-Interscience)

Stepnik, B. 2001a, Ph.D. thesis, Emission submillimétrique du milieu interstellaire: coagulation des grains, Ile de France

Stepnik, B., Abergel, A., Bernard, J.-Ph., et al. 2001b, ASPC, 243, 47

Stepnik, B., Abergel, A., Bernard, J.-Ph., et al. 2003, A\&A, 398, 551

Strong, A. W., Bloemen, J. B. G. M., Dame, T. M., et al. 1988, A\&A, 207, 1

Tielens, A. 1989, IAU Symp., 135, 239

Tielens, A. G. G. M., \& Allamandolla, L. J. 1987, ASSL, 134, 397

Tristram, M., Patanchon, G., Macías-Pérez, J. F., et al. 2005, A\&A, 436, 785

Van de Bult, C. E. P. M., Greenberg, J. M., \& Whittet, D. C. B. 1985, MNRAS, 214,289 
D. Paradis et al.: Dust emissivity variations in the Milky Way, Online Material $p 1$

Appendix: A

Table 7. Results of the IR/HI correlations in the atomic phase for all sky regions. Cols. 1-2: central position of each studied regions in degree. Column 3: number of pixels used for the correlations in each studied regions. Columns 4-11: results of the correlations in $\mathrm{MJy} / \mathrm{sr}$ for $10^{21} \mathrm{H} / \mathrm{cm}^{2}$. Columns 12-13: emissivity spectral indices in the range 100-240 $\mu \mathrm{m}\left(\beta^{\mathrm{FIR}}\right)$ and in the range 550-2100 $\mu \mathrm{m}\left(\beta^{\text {submm }}\right)$. Column 14 : derived dust temperature in Kelvin. Errors bars represent the 1- $\sigma$ uncertainty estimates.

\begin{tabular}{|c|c|c|c|c|c|c|c|c|c|c|c|c|c|}
\hline g. & at. & X. & $\begin{array}{l}a_{100} \\
\pm \Delta a_{100}\end{array}$ & $\begin{array}{l}a_{140} \\
\pm \Delta a_{140}\end{array}$ & $\begin{array}{l}a_{240} \\
\pm \Delta a_{240}\end{array}$ & $\begin{array}{l}50 \\
\Delta a_{550}\end{array}$ & $\begin{array}{l}a_{850} \\
\pm \Delta a_{850} \\
\left(\times 10^{-1}\right)\end{array}$ & $\begin{array}{l}a_{1382} \\
\pm \Delta a_{1382} \\
\left(\times 10^{-2}\right)\end{array}$ & $\begin{array}{l}a_{2098} \\
\pm \Delta a_{2098} \\
\left(\times 10^{-2}\right)\end{array}$ & $\begin{array}{l}a_{3191} \\
\pm \Delta a_{3191} \\
\quad\left(\times 10^{-3}\right)\end{array}$ & $\begin{array}{l}\beta_{\mathrm{HI}}^{\mathrm{FIR}} \\
\pm \Delta \beta_{\mathrm{HI}}^{\mathrm{FIR}}\end{array}$ & $\begin{array}{l}\beta_{\mathrm{HI}}^{\text {submm }} \\
\pm \Delta \beta_{\mathrm{HI}}^{\text {submm }}\end{array}$ & $\begin{array}{l}T_{\mathrm{d}}^{\mathrm{HI}} \\
\pm \Delta T_{\mathrm{d}}^{\mathrm{HI}}\end{array}$ \\
\hline \multicolumn{14}{|l|}{ Case 1} \\
\hline 108.34 & 3.28 & 71 & $.0 \pm 1.3$ & $4 \pm 2.3$ & $9 \pm 1.4$ & $2 \pm 0.2$ & $6 \pm 0.4$ & $.3 \pm 0.8$ & $1 \pm 0.4$ & $13.5 \pm 1.9$ & $3 \pm 0.5$ & $1.6 \pm 0.1$ & $7.2 \pm 0$ \\
\hline 117.55 & -2.16 & 111 & & & & & & & & & & & \\
\hline 120.61 & -6.34 & 40 & & & & & & & & & & & \\
\hline 139.02 & 12.46 & 17 & & & & & & & & & $2.0 \pm 3.1$ & $2.1 \pm 0.7$ & $15.7 \pm 0.6$ \\
\hline 142.09 & 2.01 & 111 & & & 1.0 & & & 12.4 & & & $2.4 \pm 0.6$ & $1.7 \pm 0.1$ & 0.5 \\
\hline 145.16 & -4.25 & 91 & & & & & & & & & & & 0.5 \\
\hline 148.23 & -6.34 & 38 & & & & & & & & & & & \\
\hline 148.23 & 2.01 & 111 & & & & & & & & & 0.4 & 0.1 & 0.5 \\
\hline 151.29 & -0.07 & 110 & & & & & & & & & $2.3 \pm 0.6$ & $0.6 \pm 0.2$ & $7.7 \pm 0.6$ \\
\hline 151.29 & 6.19 & 100 & & & & & & & & & & & \\
\hline 154.36 & -10.52 & 51 & & & & & & - & - & & & & \\
\hline 157.43 & 12.46 & 33 & & & & & & & & & & $2.9 \pm 0.6$ & 0.7 \\
\hline 160.50 & & 112 & & & & & & & & & & & \\
\hline 160.50 & 2.01 & 117 & & & & & & & & & & & \\
\hline 163.57 & & 42 & & & & & & & & & & & \\
\hline .57 & 1 & 59 & & & & & & & & & & & \\
\hline 166.63 & 8.28 & 34 & & & & & & & & .4 & & & \\
\hline 172.77 & -10.52 & 110 & & & & & & & & & & & \\
\hline Median & & & & & & & & & & & & & \\
\hline 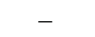 & - & - & & & & & & & & & & $6 \pm 0.3$ & $0+05$ \\
\hline \multicolumn{14}{|l|}{ Case 2 } \\
\hline 71.53 & & 64 & & & & & & & & & & & \\
\hline & & 86 & & & & & & & & & & & \\
\hline 77.66 & 4. & 104 & & & & & & & & & & & \\
\hline 73 & 8. & 56 & & & & & & & & & & & \\
\hline 83.80 & -4.25 & 59 & & & & & & & & & & & \\
\hline 89.93 & & 111 & & & & & & & & & & & \\
\hline 00 & -0.07 & 111 & & & & & & & & & & & \\
\hline 99.14 & -4.25 & 91 & & & & & & & & & & & \\
\hline & -0.07 & 110 & & & & & & & & & & & \\
\hline 14 & & 79 & & & & & & & & & & & \\
\hline & & 99 & & & & & & & & & & & \\
\hline & & 68 & & & & & & & & & & & \\
\hline & -4.25 & 90 & & & & & & & & & & & \\
\hline & & 111 & & & & & & & & & & & \\
\hline & & & & & & & & & & & & & \\
\hline & -0.07 & 110 & & & & & & & & & & & \\
\hline & & & & & & & & & & & & & \\
\hline & & & & & & & & & & & & & \\
\hline & & & & & & & & & & & & & \\
\hline & & 40 & & & & & & & & & & & \\
\hline & & 106 & & & & & & & & & & & \\
\hline & -4.25 & 62 & & & & & & & & & & & \\
\hline & & 111 & & & & & & & & & & & \\
\hline & & & & & & & & & & & & & \\
\hline & & & & & & & & & & & & & \\
\hline & & & & & & & & & & & & & \\
\hline & & 110 & & & & & & & & & & & \\
\hline & & & & & & & & & & & & & \\
\hline & & 110 & & & & & & & & & 5 & & \\
\hline & 0.96 & 72 & & & & & & & & & - & & 1.1 \\
\hline & & 91 & & & & & & & & & & & \\
\hline & & 117 & & & & & & & & & & 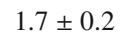 & \\
\hline & 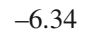 & 110 & & & & & & $<2.3$ & & & & - & 0.3 \\
\hline & & & & & & & & & & & & & \\
\hline & -14.69 & 110 & & & & & & & & & & & \\
\hline & & 91 & & & & & & 1.8 & 1.3 & & 2.2 & & $8 \pm 0.6$ \\
\hline & & 81 & & & & & & & & & & & \\
\hline & & 10 & & & & & & & & & & & $15.4 \pm 0.5$ \\
\hline & -25.05 & 51 & & & & & & & & & & & \\
\hline & 001 & 111 & $6.3 \pm 0.5$ & & & $5.4 \pm 0.5$ & $11.3 \pm 0.9$ & $25.3 \pm 1.7$ & $8.6 \pm 0.5$ & $10.6 \pm 1.5$ & $1.7 \pm 0.5$ & $1.6 \pm 0.1$ & $16.5 \pm 0.6$ \\
\hline
\end{tabular}


D. Paradis et al.: Dust emissivity variations in the Milky Way, Online Material p 2

Table 7. continued.

\begin{tabular}{|c|c|c|c|c|c|c|c|c|c|c|c|c|c|}
\hline Long. & Lat. & pix. & $\begin{array}{l}a_{100} \\
\pm \Delta \mathrm{a}_{100}\end{array}$ & $\begin{array}{l}a_{140} \\
\pm \Delta a_{140}\end{array}$ & $\begin{array}{l}a_{240} \\
\pm \Delta a_{240}\end{array}$ & $\begin{array}{l}a_{550} \\
\pm \Delta a_{550}\end{array}$ & $\begin{array}{l}a_{850} \\
\pm \Delta a_{850} \\
\quad\left(\times 10^{-1}\right)\end{array}$ & $\begin{array}{l}a_{1382} \\
\pm \Delta a_{1382} \\
\left(\times 10^{-2}\right)\end{array}$ & $\begin{array}{l}a_{2098} \\
\pm \Delta a_{2098} \\
\quad\left(\times 10^{-2}\right)\end{array}$ & $\begin{array}{l}a_{3191} \\
\pm \Delta a_{3191} \\
\quad\left(\times 10^{-3}\right)\end{array}$ & $\begin{array}{l}\beta_{\mathrm{HI}}^{\mathrm{FIR}} \\
\pm \Delta \beta_{\mathrm{HI}}^{\mathrm{FIR}}\end{array}$ & $\begin{array}{l}\beta_{\mathrm{HI}}^{\text {submm }} \\
\pm \Delta \beta_{\mathrm{HI}}^{\text {submm }}\end{array}$ & $\begin{array}{l}T_{\mathrm{d}}^{\mathrm{HI}} \\
\pm \Delta T_{\mathrm{d}}^{\mathrm{HI}}\end{array}$ \\
\hline 181.97 & 2.61 & 66 & $7 \pm 1.0$ & 3.3 & $7 \pm 2.1$ & $3 \pm 0.4$ & $8.6 \pm 0.6$ & $7.3 \pm 2.2$ & $<3.9$ & $<3.2$ & $2.2 \pm 0.9$ & $2.2 \pm 0.5$ & $15.9 \pm 0.7$ \\
\hline 181.97 & -8.43 & 48 & $2 \pm 1.2$ & & & $2 \pm 0.5$ & $8.5 \pm 0.9$ & $19.8 \pm 2.8$ & $.1 \pm 1.2$ & $3.2 \pm 5.5$ & $4.3 \pm 1.0$ & $1.7 \pm 0.3$ & $10.4 \pm 0.2$ \\
\hline 181.97 & -2.16 & 111 & $1 \pm 0.6$ & & & \pm 0.5 & $1.3 \pm 0.8$ & & $.8 \pm 0.6$ & $27.2 \pm 2.9$ & $1.9 \pm 0.5$ & $1.4 \pm 0.2$ & 0.5 \\
\hline 181.97 & 2.01 & 111 & 0.5 & & & .3 & .4 & & $7.8 \pm$ & $7.5 \pm 2.1$ & $2.0 \pm 0.4$ & $1.6 \pm 0.1$ & 16.6 \\
\hline 185.04 & -10.52 & 48 & $5.0 \pm$ & 2.6 & .9 & $0.8 \pm 0.2$ & $0.4 \pm$ & .9 & $1.4 \pm 0.9$ & - & $3.3 \pm 0.6$ & $1.9 \pm 0.3$ & $12.9 \pm 0.3$ \\
\hline Median & alues & & & & & & & & & & & & \\
\hline- & - & - & $.3 \pm 0.6$ & 1.7 & 1.3 & 0.2 & 0.6 & 1.1 & $4.1 \pm 0.5$ & $7.2 \pm 2.1$ & $2.2 \pm 0.5$ & $1.7 \pm 0.2$ & $16.9 \pm 0.6$ \\
\hline \multicolumn{14}{|l|}{ Case 3} \\
\hline & 4.10 & 34 & $7 \pm 1.5$ & 3.1 & 2.0 & \pm 0.2 & \pm 1.9 & \pm 1.3 & \pm 0.8 & $2 \pm 2.9$ & $6 \pm 0.9$ & $2.1 \pm 0.2$ & $0 \pm 0.5$ \\
\hline 77.66 & -0.07 & 61 & $38.6 \pm 10.7$ & 2.0 & 5.0 & \pm 0.4 & $9.3 \pm 2.5$ & 2.9 & $7.3 \pm 1.4$ & $8.8 \pm 2.0$ & $3.3 \pm 0.7$ & $1.7 \pm 0.3$ & 17.0 \\
\hline 77.66 & 2.01 & 107 & $.3 \pm 7.2$ & $25.4 \pm 8.2$ & & \pm 0.3 & & & $5.8 \pm 0.9$ & $8.6 \pm 1.5$ & $2.6 \pm 1.3$ & $1.6 \pm 0.2$ & 0.6 \\
\hline 83.80 & -0.07 & 105 & & 10.1 & 4.8 & \pm 0.3 & 1.3 & .3 & \pm 0.9 & $6.7 \pm 2.0$ & $.0 \pm 1.5$ & $1.7 \pm$ & 0.5 \\
\hline 93.00 & -12.61 & 17 & & & & & & & & & $.9 \pm 0.7$ & & \\
\hline 96.07 & -6.34 & 43 & $2.9 \pm 8.1$ & 0.5 & 4.8 & $2.7 \pm 0.4$ & 7.6 & & $1.1 \pm 1.3$ & $3.1 \pm$ & - & 2.4 & 14. \\
\hline 96.07 & -4.25 & 100 & & & & $5 \pm 0.2$ & & & $5.4 \pm 0.5$ & $5 \pm$ & $2.8 \pm 0.8$ & 1.2 & 0.4 \\
\hline 102.21 & -0.07 & 111 & & & & & & & 4 & & $.4 \pm 0.8$ & .1 & 0.5 \\
\hline 105.27 & -0.07 & 111 & & & & & & & $4.7 \pm$ & .2 & $.5 \pm 0.9$ & $1.5=$ & 0.4 \\
\hline 126.75 & -2.16 & 108 & .0 & & & .2 & & & $4.2 \pm$ & $7.6 \pm 1.2$ & $3.0 \pm 0.8$ & $1.2=$ & 12.8 \\
\hline 142.09 & -2.16 & 111 & 8 & 1.4 & .9 & 1 & $8.2=$ & & $3.2 \pm 0.3$ & $7.1 \pm 1.2$ & $2.1 \pm 0.5$ & $1.9 \pm 0.1$ & 16.6 \\
\hline 142.09 & 12.46 & 55 & & & & & & & $6.2 \pm 1.2$ & .5 & - & & \\
\hline 145.16 & -2.16 & 110 & & 0.8 & .7 & \pm 0.1 & & 17.2 & $3.7 \pm 0.3$ & & $2.2 \pm 0.5$ & .1 & 0.6 \\
\hline 148.23 & -2.16 & 111 & & & & & & & & & & & 15. \\
\hline & -8.43 & 53 & & & & & & & & & $6.5 \pm 1.8$ & $2.4=$ & \\
\hline .36 & -4.25 & 98 & & & & 1 & 3 & 5 & 3 & 3 & $4.3 \pm 0.4$ & $1.0 \pm$ & 0.3 \\
\hline 154.36 & 14.54 & 20 & \pm 3.2 & $34.4 \pm 14.9$ & $17.2 \pm 11.4$ & $0.9 \pm 2.8$ & $1.6 \pm 7.5$ & $7.2 \pm 13.5$ & $28.7 \pm 12.8$ & $25.5 \pm 44.8$ & $6.1 \pm 2.1$ & - & 0.3 \\
\hline 157.43 & 2.01 & 111 & & & & & & $15.7 \pm 0.7$ & & & $2.2 \pm 0.4$ & & $=0.5$ \\
\hline 157.43 & 6.19 & 73 & & & & 1 & & & $0.7 \pm 0.4$ & $<4$. & $3.5 \pm 0.7$ & & 0.3 \\
\hline 166.63 & -2.16 & 111 & \pm 0.2 & & .5 & .2 & $4.2 \pm$ & 0.5 & $2.2 \pm 0.3$ & $6 \pm 1.3$ & $2.7 \pm 0.4$ & $1.5 \pm 0.2$ & $=0.4$ \\
\hline 166.63 & 2.01 & 111 & $6.3 \pm 0.5$ & & \pm 1.0 & $2.3 \pm 0.2$ & $6.5 \pm 0.6$ & 19.4 & $6.7 \pm 0.5$ & $22.6 \pm 2.1$ & $2.1 \pm 0.5$ & $1.2 \pm 0.1$ & 16.2 \\
\hline 166.63 & 4.10 & 90 & & & & & & & & $12.3 \pm 2.1$ & & $1.3 \pm 0.2$ & \\
\hline 169.70 & -12.61 & 110 & & & & $0.5 \pm$ & $6.5=$ & 14.7 & $5.4 \pm 1.1$ & $8.9 \pm 4.4$ & $3.7 \pm 0.9$ & $1.1 \pm$ & 11.8 \\
\hline 178.91 & -6.34 & 93 & $4.0 \pm 0.8$ & & & $1.5 \pm 0.3$ & $2.6 \pm 1.5$ & $<4.8$ & $<1.2$ & - & $8.5 \pm 4.5$ & $3.8 \pm 2.0$ & $9.3 \pm 0.4$ \\
\hline 178.91 & 6.19 & 88 & $.7 \pm 0.7$ & $8 \pm 1.6$ & $6 \pm 1.1$ & $1.3 \pm 0.2$ & $6.0 \pm 0.5$ & $19.1 \pm 1.3$ & $5.7 \pm 0.7$ & $<4.9$ & $3.0 \pm 0.7$ & $1.0 \pm 0.2$ & $12.8 \pm 0.4$ \\
\hline Median & values & & & & & & & & & & & & \\
\hline- & - & - & 0.8 & $2.0 \pm 2.0$ & $1.7 \pm$ & $2.1 \pm 0.2$ & $6.5 \pm$ & 15.7 & $4.4 \pm 0.6$ & $8.8 \pm 2.0$ & $2.7 \pm 0.7$ & $1.7 \pm 0.2$ & 14.0 \\
\hline
\end{tabular}


Table 8. Results of the IR/CO correlations in the molecular phase for all sky regions. Columns 1-2: central position of each studied regions in degree. Column 3: number of pixels used for the correlations in each studied regions. Columns 4-11: results of the correlations in MJy/sr for $10^{21} \mathrm{H} / \mathrm{cm}^{2}$. Colums 12-13: emissivity spectral indices in the range $100-240 \mu \mathrm{m}\left(\beta^{\mathrm{FIR}}\right)$ and in the range 550-2100 $\mu \mathrm{m}\left(\beta^{\text {submm }}\right)$. Column 14 : derived dust temperature in Kelvin. Errors bars represent the $1-\sigma$ uncertainty estimates. Uncertainties equal to 0.0 are inferior to 0.05 .

\begin{tabular}{|c|c|c|c|c|c|c|c|c|c|c|c|c|c|}
\hline Long. & at. & pix. & $\begin{array}{l}b_{100} \\
\pm \Delta \mathrm{b}_{100}\end{array}$ & $\begin{array}{l}b_{140} \\
\pm \Delta b_{140}\end{array}$ & $\begin{array}{l}b_{240} \\
\pm \Delta b_{240}\end{array}$ & $\begin{array}{l}b_{550} \\
\pm \Delta b_{550}\end{array}$ & $\begin{array}{l}b_{850} \\
\pm \Delta b_{850} \\
\left(\times 10^{-1}\right)\end{array}$ & $\begin{array}{l}b_{1382} \\
\pm \Delta b_{1382} \\
\left(\times 10^{-2}\right)\end{array}$ & $\begin{array}{l}b_{2098} \\
\pm \Delta b_{2098} \\
\left(\times 10^{-2}\right)\end{array}$ & $\begin{array}{l}b_{3191} \\
\pm \Delta b_{3191} \\
\left(\times 10^{-3}\right)\end{array}$ & $\begin{array}{l}\beta_{\mathrm{CO}}^{\mathrm{FIR}} \\
\pm \Delta \beta_{\mathrm{CO}}^{\mathrm{FIR}}\end{array}$ & $\begin{array}{l}\beta_{\mathrm{CO}}^{\text {submm }} \\
\pm \Delta \beta_{\mathrm{CO}}^{\text {submm }}\end{array}$ & $\begin{array}{l}T_{\mathrm{d}}^{\mathrm{CO}} \\
\pm \Delta T_{\mathrm{d}}^{\mathrm{CO}}\end{array}$ \\
\hline \multicolumn{14}{|l|}{ Case 1} \\
\hline 08.34 & 8 & 71 & 1 & 0 & 2 & 2 & 3 & 7 & 4 & $9.2 \pm 1.6$ & .J & .2 & 0.6 \\
\hline 117.55 & -2.16 & 111 & & & & & & & $3 \pm 0.3$ & $10.0 \pm 1.3$ & $2.3 \pm 0.6$ & $1.2 \pm 0.2$ & $16.2 \pm 0.6$ \\
\hline 120.61 & -6.34 & 40 & \pm 6.2 & $2 \pm 7.9$ & $.4 \pm 4.1$ & 0.4 & 9 & .7 & .3 & 5.9 & $2.2 \pm 1.4$ & & 0.6 \\
\hline 139.02 & 12.46 & 17 & & $7.9 \pm 2.5$ & & $3 \pm 0.3$ & & & $0 \pm 1.2$ & $14.7 \pm 7.7$ & & & $15.1 \pm 0.5$ \\
\hline 142.09 & 2.01 & 111 & & $14.3 \pm 1.3$ & $13.0 \pm 0.8$ & & & $13.1 \pm 0.5$ & & & $2.3 \pm 0.5$ & & $15.1 \pm 0.5$ \\
\hline 145.16 & 4.25 & 91 & & 0 & 8 & 3 & & & 0.6 & & $1.8 \pm 0.6$ & & 0.5 \\
\hline 148.23 & -6.34 & 38 & & $3 \pm 1.6$ & $8 \pm 1.2$ & $=0.2$ & & 1.7 & & & $.8 \pm 0.6$ & & 0.2 \\
\hline 148.23 & 2.01 & 111 & & $9.6 \pm 0.7$ & & $6 \pm 0.1$ & & & & & $2.4 \pm 0.5$ & & $14.1 \pm 0.4$ \\
\hline 151.29 & 0.07 & 110 & & & & & & & & & .5 & & \\
\hline 151.29 & 6.19 & 100 & & & & & & & & & $.0 \pm 0.4$ & & 0.6 \\
\hline 154.36 & -10.52 & 51 & & & & & & & & & $2.4 \pm 0.4$ & & $=0.4$ \\
\hline 157.43 & 12.46 & 33 & & & & & & & & & & & 0.4 \\
\hline 160.50 & -8.43 & 112 & & & & & & & & & 0.5 & & 0.3 \\
\hline 160.50 & & 117 & & & & & & & & $5.1 \pm 1.6$ & $2.1 \pm 0.4$ & $1.6 \pm 0.2$ & $=0.4$ \\
\hline 163.57 & & 42 & & & & & & & &  & & - & \\
\hline 163.57 & 10.37 & 59 & & & & & $<$ & & $<0.4$ & - & & - & \\
\hline 166.63 & & 34 & & & & & & & & - & $6 \pm 0.7$ & - & 0.3 \\
\hline 172.77 & 0.52 & 110 & & & & & & & & & & & \\
\hline Median & vilues & & & & & & & & & & & & \\
\hline - & - & - & $6 \pm 0.3$ & $7.7 \pm 1.0$ & $.8 \pm 0.8$ & $.2 \pm 0.1$ & $1+05$ & $3.0 \pm 0.8$ & $23+04$ & $6.9 \pm 1.6$ & $2.4 \pm 0.6$ & $1.6 \pm 0.2$ & $4.9 \pm 0.4$ \\
\hline \multicolumn{14}{|l|}{ Case 2} \\
\hline 71.53 & & 64 & 423 & & 432 & & & 15 & \pm 0.8 & $3 \pm 3.2$ & .5 & \pm 0.2 & $7.7 \pm 0.7$ \\
\hline 74.59 & & 86 & 3.2 & & & & & & & & $6 \pm 0.6$ & & \\
\hline 77.66 & & 104 & & & & & & & & & $9 \pm 0.4$ & & \\
\hline 80.73 & & 56 & & & & & & & & & $.9 \pm 0.6$ & & \\
\hline 83.80 & -4.25 & 59 & & & & & & & & & 0.6 & & \\
\hline & & 111 & & & & & & & & & 0.5 & & \\
\hline & & 111 & & & & & & & & & $.4 \pm 0.5$ &  & \\
\hline 14 & -4.25 & 91 & & & & & & & $<4$. $\quad$ & & 0.5 & - & \\
\hline & & 110 & & & & & & & & & 0.7 & & \\
\hline 14 & & 79 & & & & & & & & & & & \\
\hline & & 99 & & & & & & & & & & & \\
\hline & & 68 & & & & & & & & & & & \\
\hline & -4.25 & 90 & & & & & & & & & $.2 \pm 0.5$ & & \\
\hline & & 111 & & & & & & & & & & & \\
\hline & & 02 & & & & & & & & & & & \\
\hline & & 110 & & & & & & & & & & & \\
\hline 11 & & 110 & & & & & & & & & & & 0.4 \\
\hline & & & & & & & & & & & & & \\
\hline & & 110 & & & & & & & & & 0.5 & & \\
\hline & -4.25 & 49 & & & & & & & & & 0.9 & & 0.4 \\
\hline & & 106 & & & & & & & & & & & \\
\hline & -4.2 & 62 & & & & & & & & & & & \\
\hline 13 & -0.07 & 111 & & & & & & & & & 0.7 & & 0.6 \\
\hline & & 110 & & & & & & & & & & & \\
\hline & & & & & & & & & & & & & \\
\hline & & 116 & & & & & & & & & & & 0.4 \\
\hline & & & & & & & & & & & & & \\
\hline & & & & & & & & & & & & & \\
\hline 157.43 & 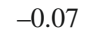 & 110 & & & & & & & & & & & $=0.5$ \\
\hline & & 72 & & & & & & & & & & & \\
\hline &  & 91 & & & & & & & & & & & \\
\hline & -0.07 & 117 & & & & & & & & & & & 0.5 \\
\hline & & 110 & & & & & & & & & & & \\
\hline & 10 & 11 & & & & & & & & & & & \\
\hline 172.77 & -14.69 & 110 & & & & & & & & & 0.5 & 0.2 & $=0.3$ \\
\hline & & 91 & & & & & & & & & & & \\
\hline & & 81 & & & & & & & & & & & \\
\hline 175.84 & & 109 & & & & & & & & & $4.3 \pm 0.4$ & 0.2 & \pm 0.3 \\
\hline & -23.05 & 51 & & & & & & & & & & & \\
\hline & & 110 & & & & & & & & & & & \\
\hline 181.97 & -12.61 & 66 & & & & & & & & & $3 \pm 2.1$ & 0.2 & \pm 0.3 \\
\hline & & 48 & & & & & & & & & 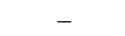 & & \\
\hline & & 11 & & & & & & & & & & & \\
\hline & & 111 & & & & & & & & & $2.9 \pm 0.5$ & & $14.0 \pm 0.4$ \\
\hline 185.04 & -10.52 & 48 & $2 \pm 0.2$ & $.6 \pm 0.8$ & $2.0 \pm 0.6$ & $<0.3$ & $2.0 \pm 0.2$ & $3.6 \pm 0.6$ & $1.1 \pm 0.3$ & $3.5 \pm 1.8$ & 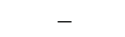 & $2.0 \pm 0.3$ & $9.8 \pm 0.2$ \\
\hline
\end{tabular}


D. Paradis et al.: Dust emissivity variations in the Milky Way, Online Material p 4

Table 8. continued.

\begin{tabular}{|c|c|c|c|c|c|c|c|c|c|c|c|c|c|}
\hline Long. & Lat. & pix. & $\begin{array}{l}b_{100} \\
\pm \Delta b_{100}\end{array}$ & $\begin{array}{l}b_{140} \\
\pm \Delta b_{140}\end{array}$ & $\begin{array}{l}b_{240} \\
\pm \Delta b_{240}\end{array}$ & $\begin{array}{l}b_{550} \\
\pm \Delta b_{550}\end{array}$ & $\begin{array}{l}b_{850} \\
\pm \Delta b_{850} \\
\quad\left(\times 10^{-1}\right)\end{array}$ & $\begin{array}{l}b_{1382} \\
\pm \Delta b_{1382} \\
\left(\times 10^{-2}\right)\end{array}$ & $\begin{array}{l}b_{2098} \\
\pm \Delta b_{2098} \\
\left(\times 10^{-2}\right)\end{array}$ & $\begin{array}{l}b_{3191} \\
\pm \Delta \mathrm{b}_{3191} \\
\quad\left(\times 10^{-3}\right)\end{array}$ & $\begin{array}{l}\beta_{\mathrm{CO}}^{\mathrm{FIR}} \\
\pm \Delta \beta_{C O}^{F I R}\end{array}$ & $\begin{array}{l}\beta_{\mathrm{CO}}^{\text {submm }} \\
\pm \Delta \beta_{\mathrm{CO}}^{\text {submm }}\end{array}$ & $\begin{array}{l}T_{\mathrm{d}}^{\mathrm{CO}} \\
\pm \Delta T_{\mathrm{d}}^{\mathrm{CO}}\end{array}$ \\
\hline Median & values & & & & & & & & & & & & \\
\hline- & - & - & $2.2 \pm 0.4$ & $6.7 \pm 0.8$ & $7.5 \pm 0.7$ & $0.9 \pm 0.1$ & $3.1 \pm 0.4$ & $8.0 \pm 0.7$ & $1.8 \pm 0.3$ & $4.9 \pm 1.3$ & $2.6 \pm 0.5$ & $1.6 \pm 0.2$ & $13.7 \pm 0.4$ \\
\hline \multicolumn{14}{|l|}{ Case 3} \\
\hline 68.46 & 4.10 & 34 & $35.1 \pm 3.4$ & $60.2 \pm 6.7$ & $34.9 \pm 4.4$ & $3.4 \pm 0.5$ & $3.4 \pm 4.2$ & $28.5 \pm 2.8$ & $6.3 \pm 1.8$ & $7.7 \pm 6.4$ & $2.7 \pm 0.5$ & $1.5 \pm 0.3$ & $17.5 \pm 0.6$ \\
\hline 77.66 & -0.07 & 61 & $27.2 \pm 2.9$ & $40.3 \pm 3.2$ & $23.8 \pm 1.3$ & $2.5 \pm 0.1$ & $8.1 \pm 0.7$ & $17.8 \pm 0.8$ & $5.4 \pm 0.4$ & $6.5 \pm 0.5$ & $2.5 \pm 0.4$ & $1.5 \pm 0.1$ & $19.6 \pm 0.8$ \\
\hline 77.66 & 2.01 & 107 & $26.3 \pm 2.9$ & $39.4 \pm 3.2$ & $23.8 \pm 1.3$ & $2.6 \pm 0.1$ & $8.7 \pm 0.6$ & $18.3 \pm 0.7$ & $5.6 \pm 0.4$ & $7.4 \pm 0.6$ & $2.5 \pm 0.4$ & $1.5 \pm 0.1$ & $19.5 \pm 0.8$ \\
\hline 83.80 & -0.07 & 105 & $42.2 \pm 3.0$ & $62.8 \pm 4.1$ & $34.5 \pm 1.9$ & $2.7 \pm 0.1$ & $10.7 \pm 0.5$ & $20.9 \pm 0.9$ & $6.7 \pm 0.3$ & $10.0 \pm 0.8$ & $2.9 \pm 0.4$ & $1.4 \pm 0.1$ & $18.0 \pm 0.7$ \\
\hline 93.00 & -12.61 & 17 & $1.9 \pm 1.3$ & $2.6 \pm 3.6$ & $5.8 \pm 2.0$ & $0.7 \pm 0.2$ & $2.9 \pm 1.1$ & $6.9 \pm 1.6$ & $2.1 \pm 1.2$ & $10.6 \pm 5.7$ & $0.9 \pm 1.9$ & $1.3 \pm 0.5$ & $20.7 \pm 0.8$ \\
\hline 96.07 & -6.34 & 43 & $11.0 \pm 3.1$ & $20.7 \pm 4.0$ & $16.7 \pm 1.8$ & $1.7 \pm 0.2$ & $5.0 \pm 0.3$ & $10.7 \pm 0.7$ & $1.3 \pm 0.5$ & $<5.9$ & $2.4 \pm 0.6$ & $1.8 \pm 0.2$ & $16.5 \pm 0.5$ \\
\hline 96.07 & -4.25 & 100 & $9.5 \pm 1.4$ & $19.2 \pm 2.0$ & $15.9 \pm 1.0$ & $2.2 \pm 0.1$ & $5.4 \pm 0.4$ & $11.2 \pm 1.0$ & $2.1 \pm 0.4$ & $0.4 \pm 1.5$ & $2.2 \pm 0.5$ & $2.0 \pm 0.2$ & $17.1 \pm 0.6$ \\
\hline 102.21 & -0.07 & 111 & $5.2 \pm 2.6$ & $10.8 \pm 3.9$ & $8.5 \pm 2.2$ & $1.4 \pm 0.2$ & $3.6 \pm 0.8$ & $8.6 \pm 1.5$ & $2.4 \pm 0.6$ & $3.9 \pm 2.7$ & $2.2 \pm 1.1$ & $1.6 \pm 0.3$ & $17.7 \pm 0.7$ \\
\hline 105.27 & -0.07 & 111 & $4.5 \pm 2.5$ & $11.8 \pm 3.6$ & $10.2 \pm 2.0$ & $2.6 \pm 0.3$ & $5.3 \pm 0.6$ & $11.8 \pm 1.1$ & $2.6 \pm 0.4$ & $5.1 \pm 1.6$ & $2.0 \pm 1.1$ & $2.0 \pm 0.2$ & $17.1 \pm 0.7$ \\
\hline 126.75 & -2.16 & 108 & $4.4 \pm 0.9$ & $7.7 \pm 1.3$ & $6.2 \pm 0.9$ & $0.5 \pm 0.1$ & $2.9 \pm 0.4$ & $6.5 \pm 0.6$ & $0.7 \pm 0.3$ & $<1.6$ & $2.5 \pm 0.6$ & $1.5 \pm 0.3$ & $16.3 \pm 0.5$ \\
\hline 142.09 & -2.16 & 111 & $3.9 \pm 1.2$ & $8.2 \pm 2.2$ & $5.7 \pm 1.5$ & $1.4 \pm 0.2$ & $1.6 \pm 0.6$ & $3.8 \pm 1.1$ & $0.3 \pm 0.5$ & $2.1 \pm 1.8$ & $2.0 \pm 0.9$ & $2.8 \pm 0.5$ & $19.5 \pm 0.9$ \\
\hline 142.09 & 12.46 & 55 & $2.1 \pm 0.2$ & $6.8 \pm 0.7$ & $6.7 \pm 0.6$ & $0.3 \pm 0.1$ & $2.0 \pm 0.7$ & $3.3 \pm 1.2$ & $1.8 \pm 0.4$ & $8.8 \pm 1.9$ & $4.4 \pm 0.5$ & $1.0 \pm 0.4$ & $11.0 \pm 0.2$ \\
\hline 145.16 & -2.16 & 110 & $3.0 \pm 0.5$ & $6.8 \pm 1.0$ & $6.3 \pm 0.9$ & $1.5 \pm 0.1$ & $3.7 \pm 0.5$ & $9.2 \pm 1.1$ & $1.3 \pm 0.4$ & $1.1 \pm 1.1$ & $1.7 \pm 0.6$ & $1.9 \pm 0.2$ & $18.4 \pm 0.7$ \\
\hline 148.23 & -2.16 & 111 & $6.0 \pm 0.6$ & $11.0 \pm 1.0$ & $9.5 \pm 0.8$ & $1.4 \pm 0.2$ & $5.2 \pm 0.5$ & $11.5 \pm 0.9$ & $2.9 \pm 0.3$ & $8.1 \pm 1.3$ & $2.0 \pm 0.5$ & $1.5 \pm 0.2$ & $18.4 \pm 0.7$ \\
\hline 154.36 & -8.43 & 53 & $0.9 \pm 0.1$ & $3.2 \pm 0.2$ & $4.1 \pm 0.3$ & $0.5 \pm 0.0$ & $1.9 \pm 0.2$ & $7.2 \pm 0.3$ & $1.4 \pm 0.2$ & $4.4 \pm 0.7$ & $2.7 \pm 0.5$ & $1.2 \pm 0.1$ & $12.6 \pm 0.3$ \\
\hline 154.36 & -4.25 & 98 & $1.1 \pm 0.2$ & $4.0 \pm 0.6$ & $5.3 \pm 0.6$ & $1.3 \pm 0.2$ & $2.7 \pm 0.4$ & $7.1 \pm 0.6$ & $1.5 \pm 0.4$ & $3.9 \pm 1.8$ & $1.9 \pm 0.6$ & $1.9 \pm 0.2$ & $14.1 \pm 0.4$ \\
\hline 154.36 & 14.54 & 20 & $2.9 \pm 0.4$ & $11.6 \pm 2.0$ & $9.9 \pm 1.6$ & $1.9 \pm 0.4$ & $2.7 \pm 1.0$ & $11.4 \pm 1.9$ & $2.3 \pm 1.8$ & $4.3 \pm 6.2$ & $3.1 \pm 0.7$ & $1.9 \pm 0.4$ & $12.8 \pm 0.5$ \\
\hline 157.43 & 2.01 & 111 & $2.3 \pm 0.2$ & $5.4 \pm 0.3$ & $5.5 \pm 0.3$ & $1.1 \pm 0.0$ & $3.5 \pm 0.2$ & $6.6 \pm 0.4$ & $1.2 \pm 0.2$ & $3.2 \pm 0.5$ & $1.8 \pm 0.4$ & $1.9 \pm 0.1$ & $17.3 \pm 0.6$ \\
\hline 157.43 & 6.19 & 73 & $2.3 \pm 0.1$ & $7.0 \pm 0.3$ & $7.4 \pm 0.3$ & $1.3 \pm 0.0$ & $4.8 \pm 0.2$ & $11.0 \pm 0.3$ & $3.0 \pm 0.1$ & $10.1 \pm 0.8$ & $2.1 \pm 0.4$ & $1.5 \pm 0.1$ & $14.7 \pm 0.5$ \\
\hline 166.63 & -2.16 & 111 & $2.7 \pm 0.3$ & $6.7 \pm 0.7$ & $7.2 \pm 0.6$ & $2.0 \pm 0.2$ & $3.6 \pm 0.5$ & $7.9 \pm 0.6$ & $1.2 \pm 0.3$ & $<3.5$ & $1.5 \pm 0.5$ & $2.3 \pm 0.2$ & $18.0 \pm 0.7$ \\
\hline 166.63 & 2.01 & 111 & $7.5 \pm 1.2$ & $16.0 \pm 3.2$ & $16.6 \pm 2.4$ & $3.9 \pm 0.5$ & $9.9 \pm 1.6$ & $21.3 \pm 2.9$ & $7.4 \pm 1.2$ & $11.9 \pm 5.3$ & $1.5 \pm 0.6$ & $1.6 \pm 0.2$ & $19.0 \pm 0.7$ \\
\hline 166.63 & 4.10 & 90 & $3.0 \pm 1.0$ & $4.4 \pm 2.6$ & $8.5 \pm 2.0$ & $1.9 \pm 0.6$ & $6.6 \pm 1.4$ & $17.5 \pm 2.5$ & $6.4 \pm 1.7$ & $17.2 \pm 5.8$ & $0.6 \pm 0.9$ & $1.1 \pm 0.3$ & $23.2 \pm 1.0$ \\
\hline 169.70 & -12.61 & 110 & $1.4 \pm 0.1$ & $5.0 \pm 0.3$ & $6.2 \pm 0.3$ & $1.3 \pm 0.0$ & $3.6 \pm 0.1$ & $7.0 \pm 0.2$ & $1.3 \pm 0.1$ & $3.8 \pm 0.5$ & $2.0 \pm 0.4$ & $2.0 \pm 0.1$ & $14.1 \pm 0.4$ \\
\hline 178.91 & -6.34 & 93 & $3.4 \pm 0.3$ & $10.6 \pm 0.7$ & $12.0 \pm 0.6$ & $1.0 \pm 0.1$ & $6.6 \pm 0.5$ & $10.9 \pm 0.5$ & $1.9 \pm 0.3$ & $0.7 \pm 1.2$ & $3.1 \pm 0.4$ & $1.8 \pm 0.2$ & $12.4 \pm 0.3$ \\
\hline 178.91 & 6.19 & 88 & $2.2 \pm 0.3$ & $5.3 \pm 0.7$ & $5.4 \pm 0.5$ & $0.9 \pm 0.1$ & $4.6 \pm 0.3$ & $7.1 \pm 0.6$ & $0.9 \pm 0.3$ & $0.3 \pm 1.1$ & $2.0 \pm 0.5$ & $1.8 \pm 0.2$ & $16.1 \pm 0.5$ \\
\hline Median & values & & & & & & & & & & & & \\
\hline- & - & - & $3.4 \pm 0.9$ & $8.2 \pm 2.0$ & $8.5 \pm 1.0$ & $1.4 \pm 0.1$ & $3.7 \pm 0.5$ & $10.7 \pm 0.9$ & $2.1 \pm 0.4$ & $4.3 \pm 1.5$ & $2.1 \pm 0.5$ & $1.6 \pm 0.2$ & $17.3 \pm 0.6$ \\
\hline
\end{tabular}

